\title{
Complex-Plane Generalization of Scalar Levin Transforms: A Robust, Rapidly Convergent Method to Compute Potentials and Fields in Multi-Layered Media
}

\author{
Kamalesh Sainath ${ }^{\mathrm{a}, *}$, Fernando L. Teixeira ${ }^{\mathrm{a}}$, Burkay Donderici ${ }^{\mathrm{b}}$ \\ ${ }^{a}$ The Ohio State University, Electroscience Laboratory, 1330 Kinnear Road, Columbus, Ohio, USA 43212 \\ ${ }^{b}$ Halliburton, Sensor Physics and Technology, 3000 N. Sam Houston Pkwy E, Houston, TX, USA 77032
}

\begin{abstract}
We propose the complex-plane generalization of a powerful algebraic sequence acceleration algorithm, the Method of Weighted Averages (MWA), to guarantee exponential-cum-algebraic convergence of Fourier and Fourier-Hankel (F-H) integral transforms. This "complex-plane" MWA, effected via a linear-path detour in the complex plane, results in rapid, absolute convergence of field/potential solutions in multi-layered environments regardless of the sourceobserver geometry and anisotropy/loss of the media present. In this work, we first introduce a new integration path used to evaluate the field contribution arising from the radiation spectra. Subsequently, we (1) exhibit the foundational relations behind the complex-plane extension to a general Levin-type sequence convergence accelerator, (2) specialize this analysis to one member of the Levin transform family (the MWA), (3) address and circumvent restrictions, arising for two-dimensional integrals associated with wave dynamics problems, through minimal complex-plane detour restrictions and a novel partition of the integration domain, (4) develop and compare two formulations based on standard/real-axis MWA variants, and (5) present validation results and convergence characteristics for one of these two formulations.
\end{abstract}

Keywords: Sommerfeld integral, Fourier integral, anisotropic media, integral convergence acceleration, Method of Weighted Averages, multi-layered environment

\section{Introduction}

In many application areas concerning time-harmonic electromagnetic (EM) fields, one encounters environments containing media of varying and arbitrary anisotropy 1 whose inhomogeneity can be approximated as multi-layered in nature. Examples include geophysical prospection [1-7], plasma physics [8], antenna design [9, 10], optical field control [11], microwave remote sensing [12], ground-penetrating radar [13, 14], and microwave circuits [15], among others. Such applications regularly encounter integrals of the form ${ }^{2}$

$$
f(\mathbf{r}) \sim \int_{-\infty}^{+\infty} \int_{f} \tilde{f}\left(k_{x}, k_{y}\right) \mathrm{e}^{i k_{x}\left(x-x^{\prime}\right)+i k_{y}\left(y-y^{\prime}\right)+i \tilde{k}_{z}\left(z-z^{\prime}\right)} \mathrm{d} k_{x} \mathrm{~d} k_{y}
$$

and/or

$$
f(\mathbf{r}) \sim \int_{-\infty}^{+\infty} \tilde{f}\left(k_{\rho}\right) H_{n}^{(1)}\left(k_{\rho}\left|\rho-\rho^{\prime}\right|\right) \mathrm{e}^{i \tilde{k}_{z}\left(z-z^{\prime}\right)} \mathrm{d} k_{\rho}
$$

\footnotetext{
${ }^{*}$ Corresponding author

Email addresses: sainath.1@osu. edu (Kamalesh Sainath), teixeira@ece. osu. edu (Fernando L. Teixeira), Burkay.Donderici@Halliburton.com (Burkay Donderici)

${ }^{1}$ We assume each medium's anisotropy manifests in diagonalizable constitutive material tensors to ensure completeness of the plane wave basis. Since all naturally-occurring media possess diagonalizable material tensors, in practical applications this assumption is always true.

${ }^{2}$ Appendix A summarizes the notation, terminology, and conventions used here.
} 
which express space-domain field/potential functions as Fourier and Fourier-Hankel (F-H) integral transforms (resp.).

In many practical applications, these integrals must often be rapidly evaluated for a wide range of longitudinal and transverse source-observer separation geometries $\mathbf{r}-\mathbf{r}^{\prime} \neq \mathbf{0}$ (e.g. for potential or field profile reconstruction). However, when using standard integration paths that run on/close to the real axis such as (1) the classic Sommerfeld Integration Path (SIP) [16] and (2) paths detouring around the branch points, branch cuts, and poles followed by real-axis integration [17-19], the convergence rate of these integrals is strongly dependent upon the transverse ( $\mathbf{r}_{t}-$ $\left.\mathbf{r}_{t}^{\prime}\right)$ and longitudinal $\left(z-z^{\prime}\right)$ separations. $\quad \mathbf{r}_{t}-\mathbf{r}_{t}^{\prime}$ determines the rapidity of the integrand's oscillation due to the Fourier and/or Hankel kernels in (1.1)-(1.2), with rising $\left|\rho-\rho^{\prime}\right|$ leading to an integrand that traditionally requires increasingly finer sampling to limit spatial aliasing and thus leads to undesirably long computation times. Furthermore, the longitudinal separation $z-z^{\prime}$ governs the rate at which the evanescent spectrum's field contribution decays with increasing transverse wave number magnitudes ${ }^{3}$, with rising $\left|z-z^{\prime}\right|$ effecting more rapid decay (and hence faster convergence) [20]. On the other hand, as $\left|z-z^{\prime}\right| \rightarrow 0$ the convergence rate lessens, with the limiting case $z-z^{\prime}=0$ yielding integrals of the form

$$
f(\mathbf{r}) \sim \int_{-\infty}^{+\infty} \int_{\tilde{f}} \tilde{f}\left(k_{x}, k_{y}\right) \mathrm{e}^{i k_{x}\left(x-x^{\prime}\right)+i k_{y}\left(y-y^{\prime}\right)} \mathrm{d} k_{x} \mathrm{~d} k_{y}
$$

and

$$
f(\mathbf{r}) \sim \int_{-\infty}^{+\infty} \tilde{f}\left(k_{\rho}\right) H_{n}^{(1)}\left(k_{\rho}\left|\rho-\rho^{\prime}\right|\right) \mathrm{d} k_{\rho}
$$

that lead to divergent results when numerically evaluated, using these standard paths, without convergence acceleration.

See Figure 1 for typical application scenarios wherein these standard paths either succeed or fail to deliver accurate field results. Observing Figure 1, one immediately realizes that devising an evaluation method for these integrals exhibiting robustness with respect to all ranges of $\mathbf{r}-\mathbf{r}^{\prime} \neq \mathbf{0}$ and medium classes (e.g. isotropic, uniaxial, biaxial) is highly desirable. This robustness criterion inherently excludes fundamentally approximate methods such as image and asymptotic methods due to their geometry-specific applicability and lack of rigorous error control [16, 17, 21, 23]. As a result, to reliably ensure accurate field results for arbitrary environmental medium composition/source-observer geometry combinations, we choose a direct numerical integration method.

In this vein, one option involves pairing standard integration methods with (real-axis path based) algebraic convergence acceleration techniques such as the standard MWA which, based on published numerical results, successfully imparts algebraic convergence acceleration even when $\left|z-z^{\prime}\right|=0$ [18, 20]. However, it is desirable to (1) guarantee absolute, exponential convergence in the classical/Riemann sense for any $\mathbf{r}-\mathbf{r}^{\prime} \neq \mathbf{0}$ separation geometry (in contrast to only guaranteeing algebraic convergence in the Abel sense when $\left|z-z^{\prime}\right|=0$ [20]) and (2) endow error control to the evanescent-zone field contribution associated with the tail integral, whose relative importance (compared to the radiation-zone contribution) to the field solution grows as $\left|\mathbf{r}-\mathbf{r}^{\prime}\right|$ decreases, to ensure that both the radiation-zone and evanescent-zone contributions are accurately evaluated ${ }^{4}$. To this end, we propose a novel numerical integration method, representing a complex-plane generalization of a specific member of the "scalar Levin-type sequence transform" (SLST) family [24] (i.e. the MWA), that:

1. bends the "extrapolation region"/tail [19] integration path sections to guarantee absolute, exponential convergence of integrals like (1.1)-(1.4),

2. imparts added, robust algebraic convergence acceleration to the tail integrals, which compounds with the exponential convergence acceleration to effect absolute, exponential-cum-algebraic convergence, via use of a linear path bend combined with our novel, complex-plane generalization of the MWA [18, 20],

3. adjusts the detour bend angles to account for the presence of branch points, branch cuts, and poles (summarily referred to here as "critical points"), and

4. addresses the added challenges associated with evaluating two-dimensional integral transforms arising as solutions to the wave equation in planar-stratified environments lacking azimuthal symmetry.

\footnotetext{
${ }^{3}$ i.e. $\left|k_{x}\right|$ and $\left|k_{y}\right|$ for Fourier double-integrals, or $\left|k_{\rho}\right|$ for F-H integrals.

${ }^{4}$ One cannot rely upon a-posteriori error checking, as was done in [18 20], for general environment/source-observer scenarios.
} 
We note that other path deformation techniques, such as the Steepest Descent Path (SDP) and one comprising the enclosure of the first/fourth quadrants of the $k_{\rho}$ plane involving an imaginary-axis integration, have been investigated and used [16, 25- 27]. However, we seek a robust integration method, valid for all $\mathbf{r}-\mathbf{r}^{\prime} \neq \mathbf{0}$ geometries, that obviates having to separately account for discrete poles while possessing applicability to multi-layered environments containing media with arbitrary anisotropy and loss. Thus while our method may result in longer solution times versus above-mentioned methods, it touts general applicability and minimal necessary book-keeping as its defining virtues.

Furthermore, a robust detour path within the pre-extrapolation region [19] maintaining a near-constant separation between the path and critical points near/on the real axis would be preferred over more traditional paths used with the MWA [17, 19]. To address this, the paper's second contribution entails a trapezoidal integration path paired with adaptive $h p$ refinement 5 .

In Section 2 we present and discuss our revision to the radiation-zone integration path 6 . In Sections 3 and 4 we develop the detoured linear integration path and complex-plane generalization to SLST for efficiently evaluating the tail sections of (1.1)-(1.4), as well as exhibit and compare two possible candidate formulations to implement the resulting modified-MWA. These developments are formulated in the context of two-dimensional integrals such as (1.1) to simultaneously address herein their additional issues versus one-dimensional integrals. However, the formulation applies equally to one-dimensional F-H transforms like (1.2) appearing in field/potential computations within cylindrically- and (azimuthal-symmetric) planar-stratified environments and, after converting the Fourier-Bessel (F-B) transform to a F-H transform [16, 28, 29], to F-B transforms as well] Section 5 presents validation results using one of the two new formulations. In Section 6 we present a study on the convergence characteristics of our algorithm as concerning the same formulation used to generate the results in Section 5 . Finally, Section 7 contains our concluding remarks.

In the ensuing discussion, we assume appropriate transformations to the material tensors and source vector have already been performed to effect a coordinate rotation such that in the resultant (azimuthal-rotated) coordinate frame, within which all integration is performed, one has $x-x^{\prime}=y-y^{\prime} \geq 08$ Discussed in detail at the end of Section 3 , this is done to guarantee absolute convergence and maximize exponential decay of both the $k_{x}$ and $k_{y}$ integrals.

\footnotetext{
${ }^{5}$ The adaptive $h p$ refinement integration methodology is the same as in [19], and thus is not discussed further here.

${ }^{6} \mathrm{We}$ present this secondary contribution first for fluidity in the narrative.

${ }^{7}$ One can accommodate the logarithmic branch-cut, manifest on the $-\operatorname{Re}\left[k_{\rho}\right]$ axis for F-H transforms [16], through a slight perturbation of the $\operatorname{Re}\left[k_{\rho}\right]<0$ half-plane path into the second quadrant.

${ }^{8}$ More generally, if $\left|x-x^{\prime}\right|=\left|y-y^{\prime}\right|$ in the rotated frame the method will work. Of course, rotating such that $x-x^{\prime} \leq 0$ forces one to alter the $k_{x}$ plane extrapolation region path such that it now incurs into the $\operatorname{Im}\left[k_{x}\right]<0$ half-plane (and similarly for $k_{y}, y-y^{\prime}$ ).
} 


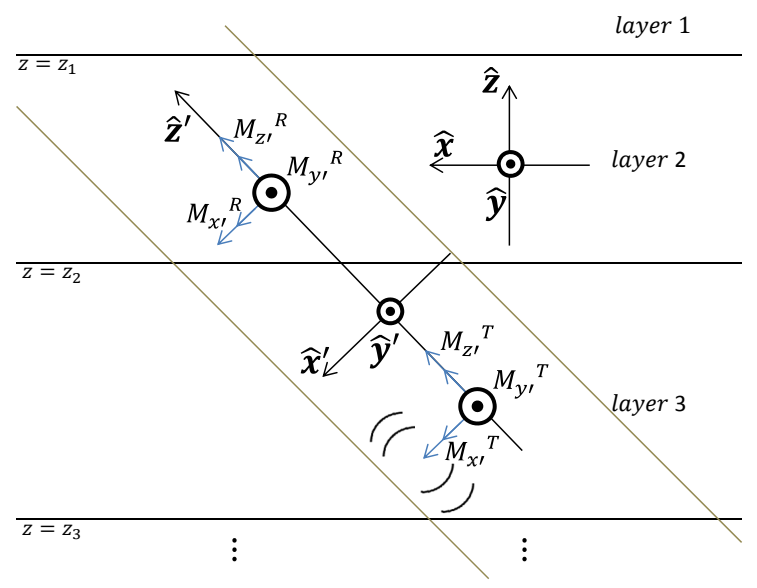

(a)

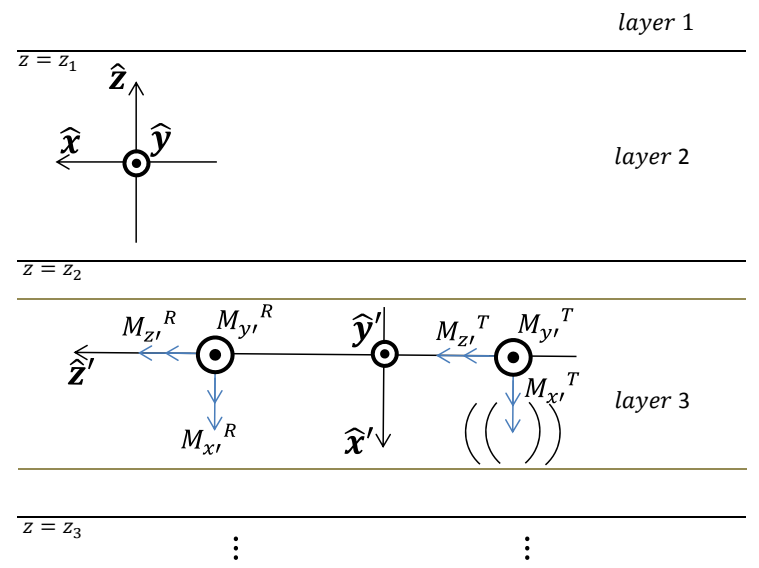

(b)

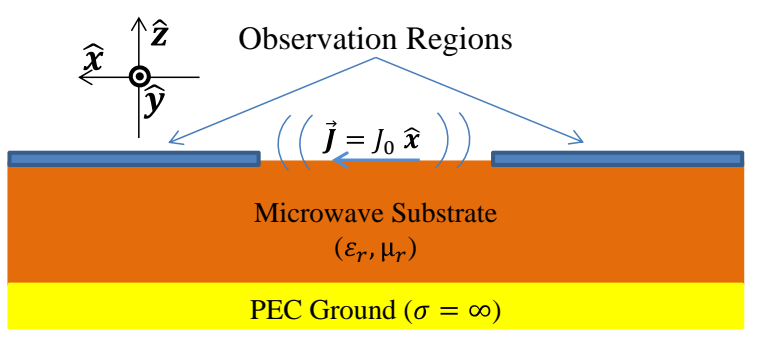

(c)

Figure 1: Figure 1a depicts a "triaxial" hydrocarbon sensor system [5] of three loop antenna transmitters $\left\{M^{T}\right\}$ and three loop antenna receivers $\left\{M^{R}\right\}$ traversing a vertical/moderately-inclined logging path bounded by a borehole (dark gold lines). Here, one typically finds $\left|z-z^{\prime}\right|$ large enough to use standard numerical integration methods, based on real-/near real-axis paths, without convergence acceleration. On the other hand, Figure $1 \mathrm{~b}$ shows the same sensor system traversing a horizontal path while Figure 1c exhibits a micro-strip geometry in which the user requests the field distribution at the air-substrate interface. The two latter geometries exhibit $0 \leq\left|z-z^{\prime}\right| \ll 1$ and represent scenarios for which these standard methods typically yield divergent results due to the oscillatory-divergent nature of integrals like 1.3 - 1.4 . 


\section{Pre-Extrapolation Region Path Revision}

First we discuss the parameterization and initial sub-division of the $k_{x}$ plane pre-extrapolation region; discussion of the $k_{y}$ plane follows identically due to our assuming $x-x^{\prime}=y-y^{\prime} \geq 0$. Applying a parameterization similar to that in [17, 19], define $\pm P_{k}$ as the points on the $\operatorname{Re}\left[k_{x}\right]$ axis within which one detours, $d_{x}$ as the maximum height of the trapezoid-shaped detour, and $\pm \xi_{1}$ as the points on the $\operatorname{Re}\left[k_{x}\right]$ axis within which one adaptively integrates (see Figure 2).

To compute $\pm P_{k}$, first define $n^{+}$as the magnitude of the real part of the global "effective" refractive index among all the layers (see [19] on computing $n^{+}$). One then computes $P_{k}$ analogously to [17] and sets $P_{k}=l_{o} k_{o}\left(n^{+}+1\right)$, where $l_{o} \geq 1$ is a user-defined pre-extrapolation region magnification constan $9^{9}$ Next, define $\Delta x=\left|x-x^{\prime}\right|=\Delta y=\left|y-y^{\prime}\right|$, $\Delta z=\left|z-z^{\prime}\right|, a^{\prime}$, and $b^{\prime}$, where $0<a^{\prime}<1 / 2$ and $b^{\prime}>1$. Now compute the following pre-extrapolation region integration path parameters [17, 19$]^{10}$

$$
\begin{aligned}
Q_{k} & =a^{\prime} k_{o}\left(n^{+}+1\right) \\
d_{x} & =\operatorname{Ln}\left(b^{\prime}\right) / \max \left(T_{0}, \Delta x\right) \\
\beta_{x} & =\tan ^{-1} \frac{d_{x}}{Q_{k}}, 0<\beta_{x}<\pi / 2 \\
\Delta \xi_{x} & =\pi / \max \left(T_{0}, \Delta x\right) \\
\xi_{1} & =\left(\operatorname{Int}\left(P_{k} / \Delta \xi_{x}\right)+1\right) \Delta \xi_{x}
\end{aligned}
$$

where $\operatorname{Int}(\cdot)$ converts its argument to an integer via fractional truncation. Now parameterize the pre-extrapolation region integration path, for $\operatorname{Re}\left[k_{x}\right]>0$, as

$$
\begin{aligned}
k_{x} & = \begin{cases}r\left(\cos \beta_{x}-i \sin \beta_{x}\right) & , 0<r<\left|Q_{k}+i d_{x}\right| \\
r-i d_{x} & , Q_{k}<r<P_{k}-Q_{k} \\
P_{k}-Q_{k}-i d_{x}+r\left(\cos \beta_{x}+i \sin \beta_{x}\right) & , 0<r<\left|Q_{k}+i d_{x}\right|\end{cases} \\
\frac{\partial k_{x}}{\partial r} & = \begin{cases}\cos \beta_{x}-i \sin \beta_{x} & , 0<r<\left|Q_{k}+i d_{x}\right| \\
1 & , Q_{k}<r<P_{k}-Q_{k} \\
\cos \beta_{x}+i \sin \beta_{x} & , 0<r<\left|Q_{k}+i d_{x}\right|\end{cases}
\end{aligned}
$$

for the trapezoidal contour (used to integrate up to $k_{x}=P_{k}$ ) combined with a real-axis path to integrate within the section $P_{k} \leq k_{x} \leq \xi_{1}$. An analogous parameterization holds for the $\operatorname{Re}\left[k_{x}\right]<0$ pre-extrapolation region path. Note that $\partial k_{x} / \partial r$ is independent of $k_{x}$ and thus can be computed prior to integration, unlike other commonly used detour 11 . This is the trapezoidal path's second benefit in addition to that mentioned in footnote 9 .

Now we splice the regions $\left(0, P_{k}\right)$ and $\left(-P_{k}, 0\right)$ each into $P$ regions, where $P$ is calculated as follows. First define

$$
d^{\prime}=\operatorname{abs}\left(\left.\frac{\partial \mathrm{e}^{i k_{x} \Delta x}}{\partial r}\right|_{r=\left|Q_{k}+i d_{x}\right|}\right)=\sin \left(\beta_{x}\right) \Delta x \mathrm{e}^{d_{x} \Delta x}
$$

as the largest magnitude assumed by $\partial \mathrm{e}^{i k_{x} \Delta x} / \partial r$ along the trapezoidal path, $c^{\prime}$ as the user-defined maximum allowed magnitude change of $\mathrm{e}^{i k_{x} \Delta x}$ between two sampling points, and $T_{1}$ and $T_{2}$ as two user-defined parameters. Subsequently, define the quantities

$$
\begin{aligned}
\Delta k_{1} & =\min \left(\pi /\left(T_{1} \max (\Delta x, \Delta z)\right), c^{\prime} / d^{\prime}\right) \\
\Delta k_{2} & =\pi /\left(T_{1} \max (\Delta x, \Delta z)\right) \\
N_{\text {node }, 1} & =\operatorname{Int}\left(\left|Q_{k}+i d_{x}\right| / \Delta k_{1}\right)+1 \\
N_{\text {node }, 2} & =\operatorname{Int}\left(\left(P_{k}-2 Q_{k}\right) / \Delta k_{2}\right)+1
\end{aligned}
$$

\footnotetext{
${ }^{9}$ This detour allows magnification of $P_{k}$ without compromising the detour height near critical points, which represents one of two primary benefits compared to the half-sine-shaped contour [17 [19].

${ }^{10} T_{0}>0$ limits the extrapolation region sub-interval length $\Delta \xi_{x}$ when $\Delta x \ll 1$ to ensure the extrapolation intervals (see Sections 23 , are adequately sampled, thereby limiting spatial aliasing.

${ }^{11}$ such as e.g. the half-sine-shaped detour [17] 19]
} 
which are used to yield $P_{m}=\operatorname{Int}\left(1+N_{\text {node, } m} / T_{2}\right)(m=1,2)$ with the corresponding final result $P=2 P_{1}+P_{2}$. Note that this method of parameterizing the pre-extrapolation region path is empirical in nature and based on the pessimistic assumption of equidistant sampling [19].

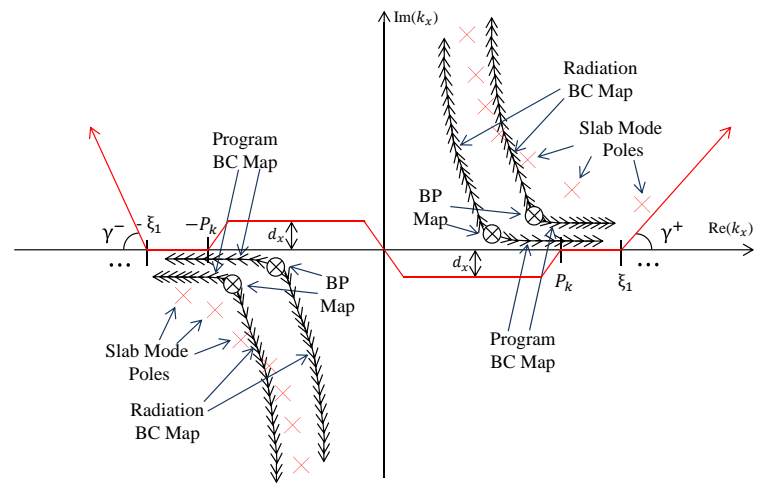

(a)

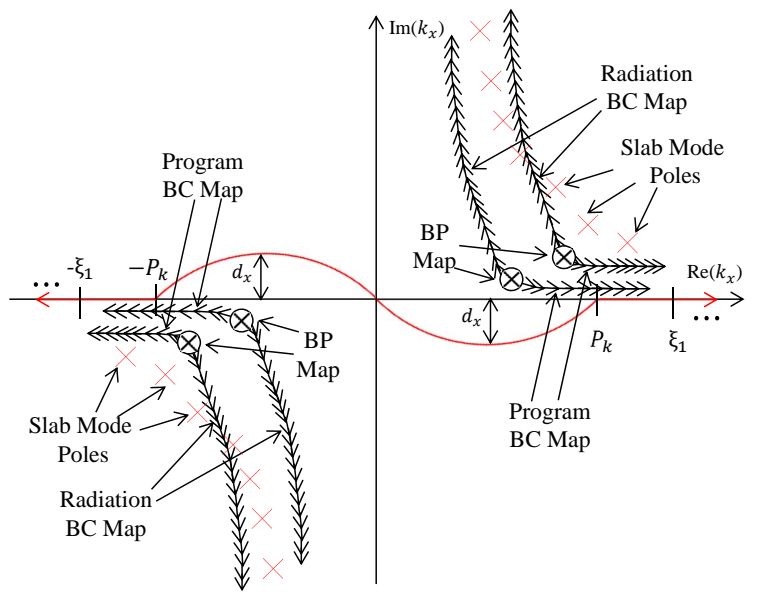

(b)

Figure 2: Figures 2a and 2b depict the new and old integration $k_{x}$ plane integration paths used in this paper and [19] (resp.). "Radiation BC Map" and "Program BC Map" refer to the branch cuts associated with the radiation/boundedness condition at infinity $\left(\operatorname{Im}\left[\tilde{k}_{z}^{2}\right]=0, \operatorname{Re}\left[\tilde{k}_{z}^{2}\right]>0\right)$ and the computer program's square root convention $\left(\operatorname{Im}\left[\tilde{k}_{z}^{2}\right]=0, \operatorname{Re}\left[\tilde{k}_{z}^{2}\right]<0\right)$ (resp.). The encircled " $\mathrm{X}$ " symbols represent branch points and the red " $\mathrm{X}$ " symbols represent guided mode poles. For $K$ extrapolation intervals used in the bottom or top method, the red contour represents the integration path connecting the end-points $k_{x}=\left(-\xi_{1}-K \Delta \xi_{x}, \xi_{1}+K \Delta \xi_{x}\right)$ or $k_{x}=\left(-\xi_{1}-t_{o}^{-} K \Delta \xi^{-^{\prime}}, \xi_{1}+t_{o}^{+} K \Delta{\xi^{+}}^{\prime}\right)$ (resp.); see Sections 23 for definitions of $\Delta \xi_{x}, \Delta \xi_{x}^{+^{\prime}}, \Delta \xi_{x}^{-^{\prime}}, t_{o}^{+}$, and $t_{o}^{-}$.

\section{Extrapolation Region Path Revision}

The MWA, initially constructed in [15, 30] with further variants developed in [18] and [20], has also demonstrated the ability to accelerate convergence of infinite-range Fourier double-integrals in high-loss, planar-stratified environments containing anisotropic media [19]. However, due to the highly oscillatory behavior of the mixed-domain integrand in integrals such as (1.1) arising from the Fourier kernels $\mathrm{e}^{i k_{x} \Delta x}$ and $\mathrm{e}^{i k_{y} \Delta y}$ when one has large $\Delta x$ and $\Delta y$ (resp.), the solution times (in our experience) became inordinately long. Therefore, it would be desirable to also deform the $k_{x}$ and $k_{y}$ plane "extrapolation" region contours to lend additional exponential decay via these two kernels, thereby dramatically accelerating convergence of the Fourier tail integrals and guaranteeing their absolute convergence even in the "worst-case" scenario $z-z^{\prime}=0$. A cursory analysis reveals an apparent severe drawback, however: one can no longer employ the MWA, which was derived assuming a real axis integration path [15, 18, 20, 30]. However, choosing 
a linear deformed path retains the MWA's algebraic convergence acceleration, as we show below ${ }^{12}$. For this analysis, take $k_{x}\left(k_{y}\right)$ as the inner (outer) integration variable.

We first exhibit the foundational relations needed to implement the complex-plane extension to a general SLST followed by exhibiting the specific case arising from modeling the tail integral truncation error using the function family stipulated in the "Mosig-Michalski Algorithm" (MMA) [18, 24]. Subsequently, we naively compute the optimal extrapolation region path detour angles without consideration for

1. the presence of critical points in the $k_{x}$ and $k_{y}$ planes and

2. two-dimensional integrals, associated with wave propagation phenomena, imparting a transitory nature to these critical points in the $k_{x}$ plane (i.e. their locations now depend on the fixed $k_{y}$ value for which the $k_{x}$ integral is evaluated).

To address the first concern, we pessimistically estimate the locations of critical points and reduce the $k_{x}$ plane departure angle of the deformed paths to ensure these features are not crossed. To address the latter concern, we (1) adjust the departure angles of the $k_{y}$ plane integration path and (2) partition the $k_{x}-k_{y}$ integration domain to ensure that the critical points

1. possess real parts with magnitude decaying as $\left|k_{y}\right|$ increases, leading to a bounded pre-extrapolation region, and

2. do not extend into the second/fourth quadrants, as this would require a) tracking their locations and b) adjusting the $k_{x}$ integration path, both of which would become functions of $k_{y}$ and lead to a non-robust integration path.

For simplicity, the analysis developing the complex-plane SLST generalization assumes isotropic planar layer 5 Fix $k_{y}$ at some (generally complex) value $k_{y 0}$ and assume $z-z^{\prime} \geq 0$, we see then that the inner integral of (1.1) writes as

$$
\int_{-\infty}^{\infty} \tilde{f}\left(k_{x}, k_{y 0}\right) \mathrm{e}^{i k_{x} \Delta x} \mathrm{e}^{i k_{y 0} \Delta y} \mathrm{e}^{i \tilde{k}_{z}^{+} \Delta z} \mathrm{~d} k_{x}
$$

where $\tilde{k}_{z}^{+}$is the up-going mode propagation constant, which for our time convention has positive imaginary part. Assuming $N$ extrapolation intervals are used [18, 19], the linear path detour used in the $k_{x}$ integration path's extrapolation region is parameterized as

$$
\begin{aligned}
k_{x} & = \begin{cases}\xi_{1}+r_{x}\left(\cos \gamma^{+}+i \sin \gamma^{+}\right) & , 0 \leq r_{x} \leq N \Delta \xi_{x}^{+^{\prime}}, \operatorname{Re}\left[k_{x}\right]>0 \\
-\xi_{1}+r_{x}\left(\cos \gamma^{-}-i \sin \gamma^{-}\right) & ,-N \Delta \xi_{x}^{\prime^{\prime}} \leq r_{x} \leq 0, \operatorname{Re}\left[k_{x}\right]<0\end{cases} \\
\frac{\partial k_{x}}{\partial r_{x}} & = \begin{cases}\cos \gamma^{+}+i \sin \gamma^{+} & , 0 \leq r_{x} \leq N \Delta \xi_{x}^{+^{\prime}}, \operatorname{Re}\left[k_{x}\right]>0 \\
\cos \gamma^{-}-i \sin \gamma^{-} & ,-N \Delta \xi_{x}^{\alpha^{\prime}} \leq r_{x} \leq 0, \operatorname{Re}\left[k_{x}\right]<0\end{cases}
\end{aligned}
$$

where one defines $\Delta \xi_{x}^{+^{\prime}}=\Delta \xi_{x} / \cos \gamma^{+}, \Delta{\xi_{x}^{-}}^{\prime}=\Delta \xi_{x} / \cos \gamma^{-},\left\{\gamma^{+}, \gamma^{-}\right\} \geq 0$, and $r_{x}$ as real-valued. $\xi_{1}$ is assumed large enough to ensure that we have sufficiently detoured past any critical points near the real axis [20]. Now recall that plane wave propagation in a homogeneous, unbounded, isotropic medium with wave number $k$ is governed by the dispersion relation $\left(\tilde{k}_{z}^{+}\right)^{2}=\left(\tilde{k}_{z}^{-}\right)^{2}=\left(k^{\prime}+i k^{\prime \prime}\right)^{2}-k_{x}^{2}-k_{y 0}^{2}$ [31]. For large $\left|k_{x}\right|$ this relation becomes ${ }^{15}$

$$
\tilde{k}_{z}^{+} \rightarrow i \begin{cases}\xi_{1}+r_{x}\left(\cos \gamma^{+}+i \sin \gamma^{+}\right) & , \operatorname{Re}\left[k_{x}\right]>0 \\ \xi_{1}-r_{x}\left(\cos \gamma^{-}-i \sin \gamma^{-}\right) & , \operatorname{Re}\left[k_{x}\right]<0\end{cases}
$$

Next, assuming that (asymptotically) $\tilde{f}\left(k_{x}, k_{y 0}\right) \rightarrow k_{x}^{q} \sum_{m=0}^{\infty} \tilde{a}_{m}\left(k_{y 0}\right) \tilde{\phi}_{m}\left(k_{x}\right)$ [18, 24], one can substitute this series expression into the extrapolation region section of 3.1) to obtain

$$
\int_{\text {ext }} k_{x}^{q} \sum_{m=0}^{\infty} \tilde{a}_{m} \tilde{\phi}_{m}\left(k_{x}\right) \mathrm{e}^{i k_{x} \Delta x} \mathrm{e}^{i k_{y 0} \Delta y} \mathrm{e}^{i \tilde{k}_{z}^{+} \Delta z} \mathrm{~d} k_{x}
$$

\footnotetext{
${ }^{12}$ The MWA is retained for robustness in field solution acceleration; the mathematical and environmental constraints present typically prevent one from integrating along the ideal Constant Phase Path, as discussed below.

${ }^{13}$ We justify this assumption based on previous analysis and results [19].

${ }^{14}$ In Sections 5.6 we demonstrate that the linear detour assures rapid convergence even in the regime $0 \leq\left|z-z^{\prime}\right| \ll 1$.

${ }^{15}$ In arriving at (3.4), the large- $\left|k_{x}\right|$ form of the dispersion relation, the proper square root sign is taken to assure exponential decay of the Fourier kernel $\mathrm{e}^{i \tilde{k}_{z}^{+} \Delta z}$ in accordance with the radiation condition.
} 
where "ext" denotes the $k_{x}$ plane extrapolation region integration path section and the $\left\{\tilde{\phi}_{m}\right\}$ comprise a family of functions used to asymptotically model $\tilde{f}\left(k_{x}, k_{y 0}\right)$ and the truncation error (discussed below) [24]. Setting $t_{o}^{+}=\cos \gamma^{+}+$ $i \sin \gamma^{+}, t_{o}^{-}=\cos \gamma^{-}-i \sin \gamma^{-}, l^{+}=t_{o}^{+} \mathrm{e}^{i k_{y 0} \Delta y} \mathrm{e}^{\xi_{1}(i \Delta x-\Delta z)}$, and $l^{-}=t_{o}^{-} \mathrm{e}^{i k_{y 0} \Delta y} \mathrm{e}^{-\xi_{1}(i \Delta x+\Delta z)}, 3.5$ becomes the union of (3.6) and 3.7):

$$
\begin{aligned}
& I_{\text {ext }}^{+}=l^{+} \sum_{m=0}^{\infty} \int_{0}^{\infty} \tilde{a}_{m} \tilde{\phi}_{m}\left(\xi_{1}+r_{x} t_{o}^{+}\right)\left(\xi_{1}+r_{x} t_{o}^{+}\right)^{q} \mathrm{e}^{r_{x} t_{o}^{+}(i \Delta x-\Delta z)} \mathrm{d} r_{x} \\
& I_{\text {ext }}^{-}=l^{-} \sum_{m=0}^{\infty} \int_{-\infty}^{0} \tilde{a}_{m} \tilde{\phi}_{m}\left(-\xi_{1}+r_{x} t_{o}^{-}\right)\left(-\xi_{1}+r_{x} t_{o}^{-}\right)^{q} \mathrm{e}^{r_{x} t_{o}^{-}(i \Delta x+\Delta z)} \mathrm{d} r_{x}
\end{aligned}
$$

with the respective truncation error integrals of 3.6 3.7 manifesting as

$$
\begin{aligned}
& I_{\mathrm{tr}}^{+}=l^{+} \sum_{m=0}^{\infty} \int_{N \Delta \xi_{x}^{+^{\prime}}}^{\infty} \tilde{a}_{m} \tilde{\phi}_{m}\left(\xi_{1}+r_{x} t_{o}^{+}\right)\left(\xi_{1}+r_{x} t_{o}^{+}\right)^{q} \mathrm{e}^{r_{x} t_{o}^{+}(i \Delta x-\Delta z)} \mathrm{d} r_{x} \\
& I_{\mathrm{tr}}^{-}=l^{-} \sum_{m=0}^{\infty} \int_{-\infty}^{-N \Delta \xi_{x}^{{ }^{\prime}}} \tilde{a}_{m} \tilde{\phi}_{m}\left(-\xi_{1}+r_{x} t_{o}^{-}\right)\left(-\xi_{1}+r_{x} t_{o}^{-}\right)^{q} \mathrm{e}^{r_{x} t_{o}^{-}(i \Delta x+\Delta z)} \mathrm{d} r_{x}
\end{aligned}
$$

Performing a change of variables on 3.8 -3.9) subsequently yields the following relations

$$
\begin{aligned}
& I_{\mathrm{tr}}^{+}=l^{+} \sum_{m=0}^{\infty} \int_{0}^{\infty} \tilde{a}_{m} \tilde{\phi}_{m}\left(\xi_{1}+\left(s+N \Delta \xi_{x}^{+^{\prime}}\right) t_{o}^{+}\right)\left(\xi_{1}+\left(s+N \Delta \xi_{x}^{+^{\prime}}\right) t_{o}^{+}\right)^{q} \mathrm{e}^{\left(s+N \Delta \xi_{x}^{+^{\prime}}\right) t_{o}^{+}(i \Delta x-\Delta z)} \mathrm{d} s \\
& I_{\mathrm{tr}}^{-}=l^{-} \sum_{m=0}^{\infty} \int_{0}^{\infty} \tilde{a}_{m} \tilde{\phi}_{m}\left(-\xi_{1}-\left(s+N \Delta \xi_{x}^{-^{\prime}}\right) t_{o}^{-}\right)\left(-\xi_{1}-\left(s+N \Delta \xi_{x}^{-^{\prime}}\right) t_{o}^{-}\right)^{q} \mathrm{e}^{-\left(s+N \Delta \xi_{x}^{-^{\prime}}\right) t_{o}^{-}(i \Delta x+\Delta z)} \mathrm{d} s
\end{aligned}
$$

Next, one evaluates 3.10)-3.11) for $M+1$ different values of $N$ (e.g. $N=1,2, \ldots, M+1)$, truncates these $M+1$ relations after the $m=(M-1)$ error series term (i.e. retain the first $M$ series terms), defines the $m$ th truncation error series coefficient pair as $\left\{\tilde{c}_{m}^{+}, \tilde{c}_{m}^{-}\right\}$(e.g. see (3.12)-(3.13) below), and solves the corresponding $(M+1)$-order system to estimate $I_{\text {ext }}^{+}$and $I_{\text {ext }}^{-}$. This procedure represents the complex-plane SLST generalization, applicable to the sequence of $M+1$ successive "cumulative tail integral" estimates [19], to accelerate evaluation of $I_{\text {ext }}^{+}$and $I_{\text {ext }}^{-}$.

Let us now examine the specific case of modeling $\tilde{f}\left(k_{x}, k_{y 0}\right)$ using the family of $(N, m)$-parameterized functions $\tilde{\phi}_{m}=\tilde{\phi}_{m}^{+}(N, s)=\left(\xi_{1}+\left(s+N \Delta \xi_{x}^{+^{\prime}}\right) t_{o}^{+}\right)^{-m}$ for $\operatorname{Re}\left[k_{x}\right]>0$ and $\tilde{\phi}_{m}=\tilde{\phi}_{m}^{-}(N, s)=\left(-\xi_{1}-\left(s+N \Delta \xi_{x}^{-^{\prime}}\right) t_{o}^{-}\right)^{-m}$ for $\operatorname{Re}\left[k_{x}\right]<0$. Performing Maclaurin expansions of the $\left\{\tilde{\phi}_{m-q}^{+}\right\}$and $\left\{\tilde{\phi}_{m-q}^{-}\right\}$, retaining only their respective zeroth-order expansion terms, setting $\omega_{N}^{+}=\exp \left(-N \Delta \xi_{x}^{+^{\prime}}\left(\cos \gamma^{+} \Delta x+\sin \gamma^{+} \Delta z\right)\right)$ and $\omega_{N}^{-}=\exp \left(-N \Delta \xi_{x}^{-^{\prime}}\left(\cos \gamma^{-} \Delta x+\sin \gamma^{-} \Delta z\right)\right)$, and defining

$$
\begin{aligned}
& \omega_{N}^{+^{\prime}}=\omega_{N}^{+} \tilde{\phi}_{-q}^{+}(N, 0), \tilde{c}_{m}^{+} \sim l^{+} \int_{0}^{\infty} \tilde{a}_{m} \mathrm{e}^{s t_{o}^{+}(i \Delta x-\Delta z)} \mathrm{e}^{i N \Delta \xi_{x}^{\prime^{\prime}}\left(\cos \gamma^{+} \Delta x-\sin \gamma^{+} \Delta z\right)} \mathrm{d} s \\
& \omega_{N}^{-^{\prime}}=\omega_{N}^{-} \tilde{\phi}_{-q}^{-}(N, 0), \tilde{c}_{m}^{-} \sim l^{-} \int_{0}^{\infty} \tilde{a}_{m} \mathrm{e}^{-s t_{o}^{-}(i \Delta x+\Delta z)} \mathrm{e}^{-i N \Delta \xi_{x}^{\prime^{\prime}}\left(\cos \gamma^{-} \Delta x-\sin \gamma^{-} \Delta z\right)} \mathrm{d} s
\end{aligned}
$$

yields a pair of expressions capturing the dominant behavior of the truncation error $I_{\mathrm{tr}}^{+} \cup I_{\mathrm{tr}}^{-}$:

$$
\begin{aligned}
& I_{\mathrm{tr}}^{+} \sim \omega_{N}^{+^{\prime}} \sum_{m=0}^{\infty} \tilde{c}_{m}^{+} \tilde{\phi}_{m}^{+}(N, 0) \\
& I_{\mathrm{tr}}^{-} \sim \omega_{N}^{-\prime} \sum_{m=0}^{\infty} \tilde{c}_{m}^{-} \tilde{\phi}_{m}^{-}(N, 0)
\end{aligned}
$$

which comprises the complex-path generalization, as concerning infinite-range Fourier integrals, to the error expression developed in Section 2 of [18]. The corresponding truncation error expressions associated with F-H transforms like (1.2) follow in analogous fashion. 
With the foundational expressions available, we now seek to maximize exponential convergence acceleration of (3.5) through a suitable choice of the detour departure angles $\gamma^{+}$and $\gamma^{-}$. Differentiating the exponent expressions $\left(\cos \gamma^{+} \Delta x+\sin \gamma^{+} \Delta z\right)$ and $\left(\cos \gamma^{-} \Delta x+\sin \gamma^{-} \Delta z\right)$ with respect to $\gamma^{+}$and $\gamma^{-}$(resp.) and setting the resulting expressions equal to zero leads us to initially (naively) choose $\gamma^{+}=\gamma^{-}=\tan ^{-1}(\Delta x / \Delta z)^{16}$, which (asymptotically) corresponds to the path of most rapid exponential decay $\sqrt{17}$ or (equivalently) the Constant Phase Path (CPP). This detour angle choice can be likened to a compromise between the so-called " $z$-transmission representation" and "radial transmission representation" [26] of the space-domain field, which were discussed therein in the context of F-H and F-B transforms. Identical expressions hold for the $k_{y}$ plane detour departure angles $\alpha^{+}$and $\alpha^{-}$. Next we consider the detour constraints imposed by these two phenomena.

To this end, first define the branch point as $k_{a}^{2}=k^{2}-k_{y 0}^{2}$ and temporarily assume that the $k_{y}$ path was chosen so that $k_{x}$ plane critical points neither manifest in the second/fourth quadrants nor migrate towards $\operatorname{Re}\left[k_{x}\right]= \pm \infty$ with increasing $\left|k_{y}\right|$. Recalling the effective refractive indices $\left\{\sqrt{\epsilon_{p m} \mu_{p n}}\right\}(m, n=1,2,3)$ for layer $p^{18}$ and how we subsequently computed $\xi_{1}$ [19], set $\gamma_{m, n, p}$ equal to either (1) the angle between $\xi_{1}$ and the $p$ th layer's $(m, n)$ th "effective wave number' $19 k_{m, n, p}=k_{o} \sqrt{\epsilon_{p m} \mu_{p n}}$, if $\operatorname{Re}\left[k_{m, n, p}\right] \geq \xi_{1}$, or (2) $\pi / 2$ if $0 \leq \operatorname{Re}\left[k_{m, n, p}\right]<\xi_{1}$. Then, $\gamma^{+}$is updated as $\gamma^{+}=\min \left(\gamma^{+},\left\{\gamma_{m, n, p}\right\}\right)$. No critical points are located in the second/fourth quadrants by assuming (for simplicity) the absence of "double-negative"/meta-material and active/gain media. Therefore, we do not have to constrain $\gamma^{-}$. However, these calculations can be readily adjusted to appropriately constrain both $\gamma^{+}$and $\gamma^{-}$if such media are present so that our assuming their absence represents a trivial constraint in our methodology.

Now we justify the assumptions above about the $k_{y}$ path, and constrain it to avoid the two issues stated earlier regarding two-dimensional integral transforms arising as the solution to wave-dynamics problems in planar-stratified environments lacking azimuthal symmetry. To this end, for some arbitrary $k_{y}$ value along the $k_{y}$ plane integration path first expand the branch point $k_{a}^{2}$ as ${ }^{20}$

$$
k_{a}^{2}=\left[\left(k^{\prime 2}-k^{\prime \prime 2}\right)-\left(k_{y}^{\prime 2}-k_{y}^{\prime \prime 2}\right)\right]+2 i\left[k^{\prime} k^{\prime \prime}-k_{y}^{\prime} k_{y}^{\prime \prime}\right]
$$

and recall that the radiation branch cut is jointly defined by the conditions $\operatorname{Im}\left[\tilde{k}_{z}^{2}\right]=0$ and $\operatorname{Re}\left[\tilde{k}_{z}^{2}\right]>0$ [16]. To ensure that critical points in the first (third) quadrant of the $k_{x}$ plane do not migrate towards $\operatorname{Re}\left[k_{x}\right]=+\infty\left(\operatorname{Re}\left[k_{x}\right]=-\infty\right)$ for large $\mid r_{y}{ }^{21}$ one must ensure that asymptotically $\operatorname{Re}\left[k_{a}^{2}\right] \rightarrow-\infty$ as $\left|r_{y}\right| \rightarrow \infty$. Observing the real part of [3.16, we see that one must constrain $\alpha^{-}$and $\alpha^{+}$to the interval $0 \leq\left\{\alpha^{-}, \alpha^{+}\right\} \leq \pi / 4$. Furthermore, to prevent critical points from migrating into the second/fourth $k_{x}$ plane quadrants, we require that $\operatorname{Im}\left[k_{a}^{2}\right] \geq 0$ as $\left|r_{y}\right| \rightarrow \infty$. Observing the imaginary part of 3.16 and noting in the region $\left\{k_{y}^{\prime}>0 \cup k_{y}^{\prime \prime}>0\right\}$ that $k_{y}^{\prime} k_{y}^{\prime \prime}>0$, a cursory analysis suggests that one cannot safely choose a non-zero value of $\alpha^{+}$without risking this migration, which would force one to dynamically re-define the $k_{x}$ integral's pre-extrapolation region path, now a function of $k_{y}$, to ensure that one (1) encloses all the quadrant one critical points that migrated into quadrant four while (2) avoiding the encirclement of quadrant three critical points that migrated into quadrant two.

As a result, it appears that one must set the additional, more restrictive constraint $\alpha^{+}=\min (0, \pi / 4)=0$, which in theory may lead to an outer integral exhibiting monotonic-divergent behavior when $0 \leq\left|z-z^{\prime}\right| \ll 1$ [20]. However, this limitation can be overcome via clever partition of the two-dimensional integration domain; see Figure 3, which summarizes the proposed partition. Integrating first over Regions I, IIa, and IIb in Figure 3 followed by integrating in Region III, which encompasses the intersection of the $k_{x}$ and $k_{y}$ plane extrapolation regions, renders the Region III integration's result immune to the migration of critical points into the second/fourth quadrants. This is because one had already stipulated a domain partitioning and completed integration over Regions I, IIa, and IIb.

We conclude that so long as one conforms to the restrictions $0 \leq\left\{\alpha^{-}, \alpha^{+}\right\} \leq \pi / 4$ and $\gamma^{+}=\min \left(\tan ^{-1}(\Delta x / \Delta z)\right.$, $\left.\left\{\gamma_{m, n, p}\right\}\right)$, one can detour in all four spectral "quadrants" $\left\{k_{x}^{\prime}>0 \cup k_{y}^{\prime}>0\right\},\left\{k_{x}^{\prime}<0 \cup k_{y}^{\prime}>0\right\},\left\{k_{x}^{\prime}>0 \cup k_{y}^{\prime}<0\right\}$,

\footnotetext{
${ }^{16}$ For F-H integrals, use the asymptotic form of the Hankel function and replace $\Delta x$ with $\left|\rho-\rho^{\prime}\right|$ when computing $\gamma^{+}=\gamma^{-}$.

${ }^{17}$ For reasons discussed in [32], this path appears similar to, but is not always necessarily, the SDP.

${ }^{18}$ These are used to compute, but are not the same as, the global effective refractive index $n^{+}$mentioned above (see [19]).

${ }^{19}$ After coercing the wave number's real part to be positive, if need be.

${ }^{20}$ One can verify that the $k_{x}$ plane discrete poles will exhibit similar behavior as the branch points [16]. Therefore, our analysis based on examining the branch point's behavior sheds analogous insight into the behavior of the poles.

${ }^{21} r_{y}$ is the $k_{y}$ plane dual of $r_{x}$, exhibited earlier.
} 
and $\left\{k_{x}^{\prime}<0 \cup k_{y}^{\prime}<0\right\}$ (see Figure 3 through which the stipulated integration path proceed ${ }^{22}$. Indeed, our proposed partition of the $k_{x}-k_{y}$ integration domain ensures that for any $\mathbf{r}-\mathbf{r}^{\prime} \neq \mathbf{0}$ geometry, the double-integral (1.1) exhibits absolute-convergent behavior in the classical/Riemann sense and thus, by Fubini's theorem [33], can be evaluated using an iterated integral whose solution is independent of the order of integration.

This discussion also brings to light the benefit of our starting assumption in this analysis, made at the end of Section 1, that $\left|x-x^{\prime}\right|=\left|y-y^{\prime}\right| \geq 0$ : a compromise is reached that ensures exponential-cum-algebraic convergence of both the $k_{x}$ and $k_{y}$ integrals throughout the integration domain. As an alternative we could have, for example, performed an azimuthal rotation such that $\left|x-x^{\prime}\right|=0$ and $\left|y-y^{\prime}\right|=\left|\rho-\rho^{\prime}\right|$ to maximize convergence acceleration of the $k_{y}$ integral. However, when $0 \leq\left|z-z^{\prime}\right| \ll 1$, the $k_{x}$ integral may exhibit monotonic-divergent behavior. In contrast to oscillatory-divergent behavior [20], the MWA variants (including the generalized version developed herein) cannot curb monotonic-divergent behavior due to the lack of oscillations that must be present for the MWA to "average out" the oscillatory-divergent sequence of cumulative tail integral estimates to obtain a final, convergent result.

Note that for integration in Regions IIa, IIb, and III in Figure 3 , one performs a separate integration and extrapolation of the individual $k_{x}$ and/or $k_{y}$ half-tail integral sections. This is in contrast to the method developed in [19] wherein we folded the half-tail integrals in the $\pm \operatorname{Re}\left[k_{x}\right]$ half-planes to yield a cosine or sine oscillatory kernel, based on assuming spectral symmetry in the environment's plane wave reflection/transmission properties, prior to performing tail integral extrapolation along the positive $\operatorname{Re}\left[k_{x}\right]$ axis (and similarly for the $k_{y}$ plane). Our present method, in bending both half-tail $k_{x}$ paths into the upper-half $k_{x}$ plane, forbids such folding due to the now-absent lack of reflection symmetry (about the $\operatorname{Im}\left[k_{x}\right]$ axis) with respect to the two halves of the extrapolation region path. The resulting penalty paid in using the complex-plane MWA manifests in having to use twice the number of weight sets versus when one can perform half-tail integral folding followed by cumulative tail integral sequence extrapolation, leading to increased memory requirement and computation time in regards to procuring the MWA weight sets. As a practical consideration, then, we wish to reduce the number of extrapolation weight sets that must be evaluated 23 . To this end, we take two steps to halve this number to the six weight sets originally required when performing folding followed by extrapolation. First, we set $\alpha^{+}=\gamma^{+}$and $\alpha^{-}=\gamma^{-}$. Second, we make the approximation (for each field component) that the asymptotic monomial power dependence on both $\left|k_{x}\right|$ and $\left|k_{y}\right|$ [31, 34] equals the average monomial power dependencies on $k_{x}$ and $k_{y}$. For example, if we determine the integrand for one field component has asymptotic monomial dependencies of $O\left(k_{x}^{q_{1}}\right)$ and $O\left(k_{y}^{q_{2}}\right)$, then we take (as both our $k_{x}$ and $k_{y}$ monomial dependence factors) $q_{o}=\operatorname{Nint}\left(\left(q_{1}+q_{2}\right) / 2\right)$, where $\operatorname{Nint}(\cdot)$ converts its argument to an integer via rounding. Furthermore, to ensure stability of the accelerator weight expressions and minimize aliasing effects due to inordinately long extrapolation region intervals, we neglect the integrand oscillation due to $\Delta z$ in the exponential kernels of $\sqrt{3.12})-(3.13)$. This allows one to update the truncation error estimates $\left\{\omega_{N}^{+^{\prime}}, \omega_{N}^{-^{\prime}}\right\}$ as $\omega_{N}^{ \pm^{\prime}}=(-1)^{N} \omega_{N}^{ \pm^{\prime}}$ when $\Delta x>T_{0}$ [18].

Beyond the concern of weight computation stability, we also ignore the phase variation associated with $\Delta z$ due to $\Delta z$, in general, being ill-defined. Indeed, in (1) an anisotropic homogeneous environment or (2) a stratified environment containing isotropic and/or anisotropic media, several phenomena typically obfuscate a univocal, clear definition for the effective longitudinal distance traversed by the characteristic plane wave fields when traveling from $\mathbf{r}^{\prime}$ in layer $M$ to $\mathbf{r}$ in layer $L$. These are (1) multi-bounce within slab layers, (2) the layer and (for anisotropic media) mode dependence of the longitudinal propagation constants, (3) interface reflections in layer $L$ causing both up-going and down-going modal fields (four total modes in general) to contribute to the observed field at $\mathbf{r}$, and (4) inter-mode coupling at the interfaces. In fact these four considerations, along with the inherently asymptotic nature of the CPP parameterization and the constraints associated with critical points/two-dimensional integrals addressed above, lead one in practice to not integrate exactly along the CPP. As a result, one typically finds the integrands of extrapolationregion integrals still exhibiting undesirable residual oscillation due to the complex exponential factors. While, for $\Delta x \geq 0$, one still always has a non-zero detour angle for both the $k_{x}$ and $k_{y}$ extrapolation region paths ${ }^{25}$ these practical

\footnotetext{
${ }^{22}$ Due to our assuming $x-x^{\prime}=y-y^{\prime} \geq 0$, it is implicitly understood that in all four "quadrants" $\operatorname{Im}\left[k_{x}\right] \geq 0$ and $\operatorname{Im}\left[k_{y}\right] \geq 0$ (excepting the minor pre-extrapolation region detour made into quadrant four).

${ }^{23}$ Nominally, there are twelve weight sets one must pre-compute and store to implement the complex-plane MWA: three field components, each with differing combinations of $\left(k_{x}, k_{y}\right)$ monomial power dependencies, multiplied by up to four distinct extrapolation region detour angles $\alpha^{+}, \alpha^{-}, \gamma^{+}$, and $\gamma^{-}$.

${ }^{24}$ Stability and aliasing considerations also motivated our choice of the break-point spacings $\Delta \xi_{x}, \Delta \xi_{x}^{+^{\prime}}$, and $\Delta \xi_{x}^{{ }^{\prime}}$.

${ }^{25}$ Recall from [19] that the pre-extrapolation region serves to detour around those critical points within a certain distance from the real axis. Therefore, the presence of critical points cannot force $\gamma^{+}$and $\gamma^{-}$to equal zero exactly.
} 
considerations are what demand the inclusion of an algebraic convergence accelerator like the MWA that exactly acts upon the very types of oscillatory integrals that will typically result. Therefore, while one does not typically realize the ideal situation of maximized exponential convergence acceleration (the strongest acceleration theoretically available here outside of the SDP), we largely mitigate this pitfall with the robust algebraic acceleration afforded by the MWA, which is agnostic to the environment/source-observer scenario (so long as $\Delta x>0$ ). Indeed, for an order- $N$ MWA method used (see below) one realizes a reduction in truncation error between $O\left(k_{x}^{-N}\right)$ and $O\left(k_{x}^{-2 N}\right)[18]$.

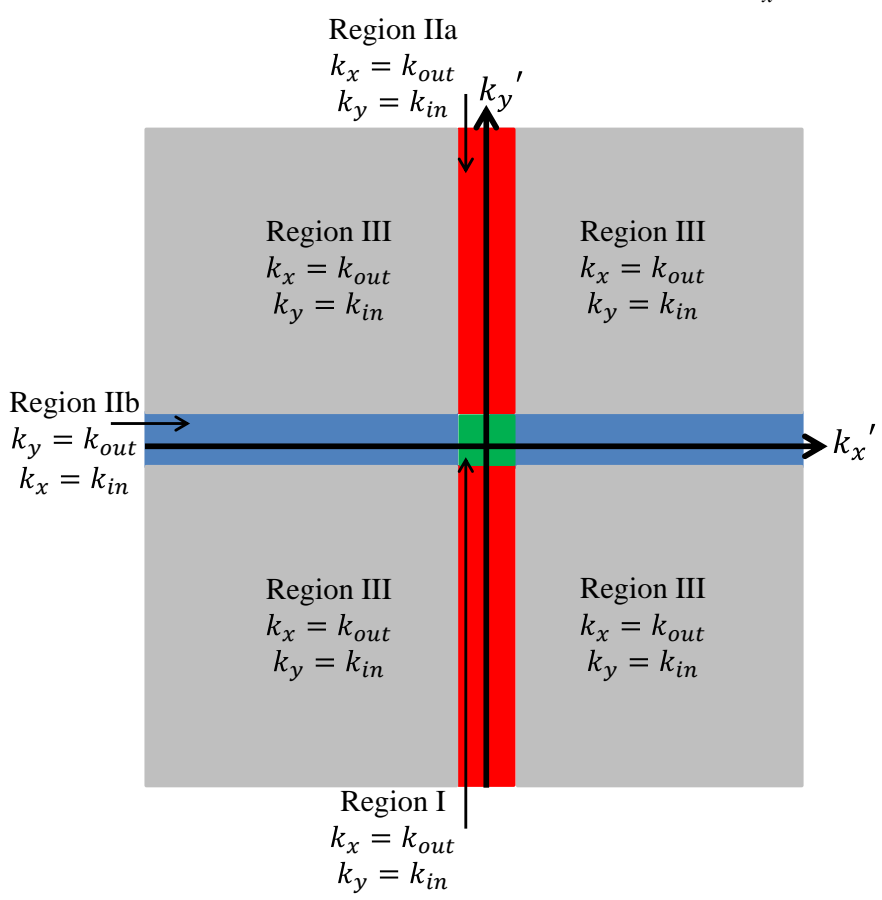

Figure 3: Depiction of the proposed integration domain partition scheme to ensure absolute convergence of Fourier doubleintegrals such as 1.1 and 1.3 . $k_{\text {out }}$ and $k_{\text {in }}$ represent the outer and inner integration variables (resp.).

\section{Revised Accelerator Weight Computation}

The MWA, both in its form as the MMA [18, 24] and its more recent variant the new/"revisited" MWA [20], each offer different, desirable attributes. The latter version offers a straightforward methodology to unambiguously define arbitrary-order accelerator weight sets and recursively compute higher-order weight sets upon demand. While we showed previously [19] for the MMA how one can reduce the FLOF ${ }^{26}$ count involving the cumulative integrals themselves, the weight computations (1) depended on whether the series of successive extrapolation region subinterval integrals exhibited oscillating or monotone behavior [18, 19], and (2) the FLOP count to compute the weight sets rapidly grows for successive weight sets, placing a practical limit on obtainable accuracy in the weights (and thus the estimated tail integral) due to roundoff error accumulation in the computed weights. On the other hand, the computation of the new MWA weight sets is (1) a numerically unstable process rapidly leading to numerical overflow (when using the form exhibited in [20]) and (2) directly linked to procuring the estimated tail integral [20], which is the solution to a highly ill-conditioned linear system (shown in Section 4.2), which previously led us to use the MMA in [19]. Nevertheless, both flavors of MWA offer useful mathematical developments for the weights that are couched in the framework of SLST, using a family of functions in a series representation to model the spectral portion of the

\footnotetext{
${ }^{26}$ Floating Point Operations
} 
mixed-domain Green's Function ${ }^{27}$ and resulting tail integral truncation error. For the MMA, the proposed series [18]

$$
\tilde{f}\left(k_{x}, k_{y 0}\right) \sim k_{x}^{q} \sum_{m=0}^{\infty} \frac{\tilde{a}_{m}\left(k_{y 0}\right)}{k_{x}^{m}}
$$

is intuitive in its form, and we confirm below the validity (and in fact optimality) of using this approximating series by a straightforward mathematical analysis 28 . It will be shown that this optimality arises due to the error modeling series (see (3.14)-(3.15) above and [18]) being entirely consistent with the closed-form expression of the truncation error both in the absence and (via linear superposition) presence of stratified inhomogeneity. The practical consequence of this function family's modeling optimality manifests in minimizing the number of cumulative tail integral estimates required to accurately estimate $I_{\text {ext }}^{+}$and $I_{\text {ext }}^{-}$, as demonstrated in Section 6

In summary, we seek a revised, complex-plane MWA that combines the best aspects of both the MMA [18] and more recent MWA variant [20], in tandem with incorporating the added exponential convergence acceleration afforded by bending the extrapolation region integration path, to effect robust and powerful field solution convergence acceleration. To this end, in this section we (1) analyze and justify using (4.1) as the approximating series for $\tilde{f}\left(k_{x}, k_{y 0}\right)$ and (2) exhibit and compare two proposed formulations for implementing the complex-plane generalization of the MWA, using the new "remainder estimates" $\left\{\omega_{N}^{+^{\prime}}, \omega_{N}^{-\prime}\right\}[18]$ and the asymptotic series expansion [4.1).

\subsection{The Optimal Error-Modeling Function Family}

Herein we examine the inner spectral $\left(k_{x}\right)$ integral for some fixed $k_{y}=k_{y 0}$ in the region $\operatorname{Re}\left[k_{x}\right]>0$. Furthermore, assume $\tilde{f}\left(k_{x}, k_{y 0}\right)$ has an asymptotic $k_{x}$ monomial dependence of $k_{x}^{q}$ [18, 20]. One then has the asymptotic truncation error

$$
I_{\mathrm{tr}}^{+}=\omega_{N}^{+} I_{\mathrm{tr}}^{+^{\prime}}=\omega_{N}^{+} \int_{0}^{\infty}\left[\xi_{1}+\left(s+N \Delta \xi_{x}^{+^{\prime}}\right) t_{o}^{+}\right]^{q} \mathrm{e}^{s t_{o}^{+}(i \Delta x-\Delta z)} \mathrm{d} s
$$

which just equals (3.10) with the asymptotic series expansion for $\tilde{f}\left(k_{x}, k_{y 0}\right)$ replaced by the dominant series term $k_{x}^{q}$. Next note that $I_{\mathrm{tr}}^{+^{\prime}}$ has a closed-form, convergent solution for $\operatorname{Re}\left[t_{o}^{+}(i \Delta x-\Delta z)\right]<0$; setting $\tilde{a}=\tilde{a}(N)=\tilde{\phi}_{0}^{+}(N, 0)=$ $\xi_{1}+t_{o}^{+} N \Delta \xi_{x}^{+^{\prime}}$ and $\chi=-t_{o}^{+}(i \Delta x-\Delta z)$, one obtains

$$
I_{\mathrm{tr}}^{+}=\omega_{N}^{+}{I_{\mathrm{tr}}^{+^{\prime}}=\omega_{N}^{+}}^{+} \begin{cases}1 / \chi & , q=0 \\ \left(t_{o}^{+}+\tilde{a} \chi\right) / \chi^{2} & , q=1 \\ \left(2 t_{o}^{+2}+2 \tilde{a} t_{o}^{+} \chi+\tilde{a}^{2} \chi^{2}\right) / \chi^{3} & , q=2\end{cases}
$$

and so on for other values of $q{ }^{29}$ Examining the asymptotic limit for these three illustrative cases, we find:

$$
\lim _{|\tilde{a}| \rightarrow \infty} I_{\mathrm{tr}}^{+^{\prime}}= \begin{cases}1 / \chi & , q=0 \\ \tilde{a} / \chi & , q=1 \\ \tilde{a}^{2} / \chi & , q=2 \\ \tilde{a}^{q} / \chi & , q \in \mathcal{N}^{+}\end{cases}
$$

where $\mathcal{N}^{+}$represents the set of positive natural numbers. Similarly, one expects that the reflected/transmitted field terms will also have an asymptotic monomial dependence [20]. For example, consider a two-layer, planar-stratified environment containing isotropic media. The $\mathrm{TE}_{z} / \mathrm{TM}_{z}$ reflection and transmission coefficients for a plane wave, incident from half-space number one upon half-space number two, write as [16]

$$
R_{\mathrm{TM}}=\frac{\epsilon_{2, r} \tilde{k}_{1, z}^{+}-\epsilon_{1, r} \tilde{k}_{2, z}^{+}}{\epsilon_{2, r} \tilde{k}_{1, z}^{+}+\epsilon_{1, r} \tilde{k}_{2, z}^{+}}, R_{\mathrm{TE}}=\frac{\mu_{2, r} \tilde{k}_{1, z}^{+}-\mu_{1, r} \tilde{k}_{2, z}^{+}}{\mu_{2, r} \tilde{k}_{1, z}^{+}+\mu_{1, r} \tilde{k}_{2, z}^{+}}, T_{\mathrm{TM}}=1+R_{\mathrm{TM}}, T_{\mathrm{TE}}=1+R_{\mathrm{TE}}
$$

\footnotetext{
${ }^{27}$ i.e. the fundamental spectral kernel embedded in the integrands of (1.1)- 1.4 .

${ }^{28}$ When assuming the spectral portion of the integrands in (1.1)- 1.4 asymptotically behave as monomial powers of $k_{x}$ and $k_{y}$, the new MWA's error-modeling functions reduce to this function family too [20].

${ }^{29}$ For the multi-layered scenario, use 3.10 and invoke superposition of the closed-form results for different monomial powers $q-m$ ( $m=$ $0,1,2, \ldots)$.
} 
where

$$
\left(\tilde{k}_{m, z}^{+}\right)^{2}=\left(\tilde{k}_{m, z}^{-}\right)^{2}=k_{o}^{2} \epsilon_{m, r} \mu_{m, r}-k_{\rho}^{2}(m=1,2), \lim _{\left|k_{\rho}\right| \rightarrow \infty} \tilde{k}_{z}^{+}=i k_{\rho}
$$

Indeed, we see that for large $\left|k_{\rho}\right|$ the reflection/transmission coefficients have a monomial power dependence $O\left(k_{\rho}^{0}\right)$.

Pulling out the dominant monomial term $\tilde{a}^{q}$ in 4.3, setting the $N$ th cumulative remainder estimate as $\omega_{N}^{+^{\prime}}=\omega_{N}^{+} \tilde{a}^{q}$, and invoking superposition (see footnote 29, one now has $\omega_{N}^{+} I_{\mathrm{tr}}^{+^{\prime}}=\omega_{N}^{+^{\prime}} \sum_{m=0}^{q} \tilde{b}_{m}^{+} / \tilde{a}^{m}$, which recovers the dominant contribution to the complex-path extension (3.14) of the error expression derived (using (4.1)) in [18] The same procedure shown above, using instead $t_{o}^{-}, \tilde{a}=\tilde{\phi}_{0}^{-}(N, 0)=-\xi_{1}-t_{o}^{-} N \Delta \xi_{x}^{-^{\prime}}$, and $\chi=t_{o}^{-}(i \Delta x+\Delta z)$, can be repeated for the $\operatorname{Re}\left[k_{x}\right]<0$ tail integral to obtain a dual set of expressions that recover (3.15). Based on this analysis, when proposing two revised MWA methods we will use the $(N, m)$-parameterized function families $\left\{\tilde{\phi}_{m}^{+}(N)=\tilde{\phi}_{m}^{+}(N, 0)\right\}$ and $\left\{\tilde{\phi}_{m}^{-}(N)=\tilde{\phi}_{m}^{-}(N, 0)\right\}$ to model the tail integral truncation error.

\subsection{Two Proposed Formulations}

For the first formulation we take inspiration from [20]. To this end, for the $\operatorname{Re}\left[k_{x}\right]>0$ tail integral first define $I_{N}^{+^{\prime}}$ and $I_{N+1}^{+^{\prime}}$ as two input cumulative tail integral estimates and the under-determined linear system, with respect to which the non-truncated tail integral $I_{\mathrm{ext}}^{+}$is defined, as [20, 24]

$$
\begin{aligned}
& I_{\mathrm{ext}}^{+}=I_{N}^{+^{\prime}}+\omega_{N}^{+^{\prime}} \sum_{m=0}^{\infty} \frac{\tilde{b}_{m}^{+}}{\tilde{a}(N)^{m}} \\
& I_{\mathrm{ext}}^{+}=I_{N+1}^{+^{\prime}}+\omega_{N+1}^{+^{\prime}} \sum_{m=0}^{\infty} \frac{\tilde{b}_{m}^{+}}{\tilde{a}(N+1)^{m}}
\end{aligned}
$$

whose equations are subsequently truncated after the $m=0$ term [20]. This truncation yields a second-order linear system solved for an improved estimate $I_{N}^{+(2)}$ of $I_{\mathrm{ext}}^{+}$that is free of the $\tilde{a}^{0}$ term in its truncation error series [18, 20, 24]:

$$
\eta_{N}^{+(1)}=-\frac{\omega_{N}^{+^{\prime}}}{\omega_{N+1}^{+^{\prime}}}, \quad I_{N}^{+(2)}=\frac{I_{N}^{+^{\prime}}+\eta_{N}^{+(1)} I_{N+1}^{+^{\prime}}}{1+\eta_{N}^{+(1)}}=I_{\mathrm{ext}}^{+}+\omega_{N}^{+^{\prime}} \frac{\sum_{m=1}^{\infty} \tilde{b}_{m}^{+}\left[\tilde{a}(N+1)^{-m}-\tilde{a}(N)^{-m}\right]}{1+\eta_{N}^{+(1)}}
$$

Similarly, using $M+1(M=1,2, \ldots)$ cumulative tail integral estimates $\left\{I_{1}^{+^{\prime}}, I_{2}^{+^{\prime}}, \ldots, I_{M+1}^{+^{\prime}}\right\}$ to eliminate the first $M$ terms of $\sum_{m=0}^{\infty} \tilde{b}_{m}^{+} / \tilde{a}^{m}$, one has for the $P$ th truncated linear equation $(P=1,2, \ldots, M+1) I_{1}^{+(M+1)}=I_{P}^{+^{\prime}}+\omega_{P}^{+^{\prime}} \sum_{m=0}^{M-1} \tilde{b}_{m}^{+} / \tilde{a}(P)^{m}$. Subsequently, one procures the weights via solving the associated order- $(M+1)$ linear system for the best $I_{\text {ext }}^{+}$estimate (i.e. $I_{N}^{+(M+1)}$ ), whose solution implicitly contains the expressions for the weights [20]. However, obtaining all desired weight tier sets by directly solving the associated linear systems (1) is very costly and (2) possibly exacerbates weight accuracy degradation due to the poor conditioning of these systems (see below). Instead, one can obtain closed-form solutions to the weight sets using the methodology outlined in [20] as adapted to our choice of (1) error-modeling functions $\left\{\tilde{a}(N)^{-m}\right\}$ and (2) truncation error estimates $\left\{\omega_{N}^{++^{\prime}}\right\}$. In [20] it was assumed that $\tilde{f}\left(k_{x}, k_{y 0}\right)$ asymptotically exhibited a monomial power dependence of the form $C k_{x}^{q}$ ( $C$ being some constant), with the obvious consequence that $\partial^{n} \tilde{f}\left(k_{x}, k_{y 0}\right) / \partial^{n} k_{x}$ corresponds to a new function asymptotically behaving as $\sim k_{x}^{q-n}[1]$ Rearranging the order- $(M+1)$ linear system thus yields a similar (but not yet identical) system to equation (22) in [20]:

$$
\left[\begin{array}{ccccc}
-1 / \omega_{1}^{+^{\prime}} & 1 & \tilde{a}(1)^{-1} & \cdots & \tilde{a}(1)^{-(M-1)} \\
-1 / \omega_{2}^{+^{\prime}} & 1 & \tilde{a}(2)^{-1} & \cdots & \tilde{a}(2)^{-(M-1)} \\
\vdots & \vdots & \vdots & \vdots & \vdots \\
-1 / \omega_{M+1}^{+^{\prime}} & 1 & \tilde{a}(M+1)^{-1} & \cdots & \tilde{a}(M+1)^{-(M-1)}
\end{array}\right]\left[\begin{array}{c}
I_{1}^{+(M+1)} \\
\tilde{b}_{o}^{+} \\
\tilde{b}_{1}^{+} \\
\vdots \\
\tilde{b}_{M-1}^{+}
\end{array}\right]=-\left[\begin{array}{c}
I_{1}^{+^{\prime}} / \omega_{1}^{+^{\prime}} \\
I_{2}^{+^{\prime}} / \omega_{2}^{+^{\prime}} \\
I_{3}^{+^{\prime}} / \omega_{3}^{+^{\prime}} \\
\vdots \\
I_{M+1}^{+^{\prime}} / \omega_{M+1}^{+^{\prime}}
\end{array}\right]
$$

Noting that the weight sets in [20] were computed for arbitrary monomial power dependence $k_{x}^{q}$, one can crossmultiply the $\tilde{a}^{q}$ factors in the $\left\{\omega_{p}^{+^{\prime}}\right\}$ across the respective rows of 4.10 to obtain an analogous system, where now the

\footnotetext{
${ }^{30}$ The coefficients $\left\{\tilde{b}_{m}^{+}\right\}$, however, are computed exactly; contrast this to the $\left\{\tilde{c}_{m}^{+}\right\}$of 3.14 .

${ }^{31}$ Examining [16 34] confirms the asymptotic monomial power dependence of $\tilde{f}\left(k_{x}, k_{y 0}\right)$.
} 
$\left\{\tilde{a}(p)^{(q-n)}\right\}$ factors in the modified form of (4.10) represent (up to a constant) successive $s$ derivatives of $\left(\xi_{1}+t_{o}^{+} s\right)^{q}$ evaluated at $s=032$. Having now matched our linear system to [20], the $n$th weight $(n=1,2, \ldots, M+1)$ for the tier- $(M+1)$, complex-plane generalization of the new MWA writes as [20]

$$
w_{n}^{(M+1)}=(-1)^{n+1}\left(\begin{array}{c}
M \\
n-1
\end{array}\right) \tilde{a}(n)^{M-1-q} / \omega_{n}^{+}
$$

with the expression for our best tail integral estimate given as

$$
I_{1}^{+(M+1)}=\frac{\sum_{n=1}^{M+1} w_{n}^{(M+1)} I_{n}^{+^{\prime}}}{\sum_{n=1}^{M} w_{n}^{(M+1)}}
$$

The expressions for the $\left\{w_{n}^{(M+1)}\right\}$ corresponding to $\operatorname{Re}\left[k_{x}\right]<0$ tail integral follows analogously. Furthermore, one expects that with a different choice of $\left\{\tilde{\phi}_{m}(N, s)\right\}$, this derivation can be repeated to develop complex-plane extensions to other SLST algorithmic members.

From an analytic standpoint, the derivation of the weights for this formulation is complete. However, despite the analytic form of the new MWA weights shown in (4.11] and [20], in a finite-precision, numerical implementation this casting leads to arithmetic overflow. This drawback, along with the numerically unstable means to recursively update the weights to procure higher-order weight sets, can be easily remedied as follows:

1. Starting at some tier- $N$ weight set (e.g. set $N=M+1$ ), multiply all the weights by $\omega_{N}^{+} / \tilde{a}(N)^{N-2-q}$. This ensures that the weights remain bounded for all $n$ and $N$.

2. To subsequently obtain a tier- $(N+1)$ weight set from the tier- $N$ set:

(a) Set $w_{N+1}^{(N+1)}=(-1)^{N+2} / N$.

(b) For the remaining $N$ weights, set $w_{n}^{(N+1)}=d_{n, N} w_{n}^{(N)}$

where

$$
d_{n, N}=\frac{e^{-\Delta \xi_{x}^{\prime^{\prime}}\left(\Delta x \sin \gamma^{+}+\Delta z \cos \gamma^{+}\right)}}{N-n+1}\left(\frac{a(N)}{a(N+1)}\right)^{N-2-q} \frac{a(n)}{a(N+1)}
$$

The second proposed formulation employs the MMA [18], as extended to facilitate adaptive tail integral evaluation [19], in conjunction with our complex extension to the truncation error estimates $\left\{\omega_{p}^{+^{\prime}}, \omega_{p}^{-^{\prime}}\right\}$. The formulae to compute arbitrary-order weight sets is given in [18], while the method to recursively find higher-order weight sets is exhibited in [19]. Therefore, the reader is referred to these two references for the elementary details.

Between these two formulations, we opt to implement and show validation results for the second formulation based on the MMA. This is because of the first formulation's poor suitability for an adaptive tail integral evaluation scheme, which in turn is due to increasingly higher-order weight sets being the solutions to increasingly ill-conditioned linear systems. Even though we now have available the analytically recast, numerically stable, closed-form expressions for the first formulation's weights and their update scheme (which obviates any potential algorithmic instability exacerbating computed weight errors), the relative accuracy of the computed weights is still fundamentally capped by the linear system's conditioning ${ }^{33}$ To illustrate the ill-conditioning of the weight computation, we show in Figure 4 below, for four different $\mathbf{r}-\mathbf{r}^{\prime}$ geometries, the two-norm condition number $C N$ [35] of [4.10] as a function of its rank $M^{\prime}=M+134$

1. $\left(x-x^{\prime}, z-z^{\prime}\right)=(1,0) \mathrm{m}$

2. $\left(x-x^{\prime}, z-z^{\prime}\right)=(1000,0) \mathrm{m}$

3. $\left(x-x^{\prime}, z-z^{\prime}\right)=(1,10) \mathrm{m}$

4. $\left(x-x^{\prime}, z-z^{\prime}\right)=(1000,10) \mathrm{m}$

\footnotetext{
${ }^{32}$ One does not have the $q$-dependent constants in columns three to $M+1$ of the matrix in 4.10 . However, one can include these constants and only affect the unneeded coefficients $\left\{\tilde{b}_{1}^{+}, \ldots, \tilde{b}_{M-1}^{+}\right\}$[35].

${ }^{33}$ Indeed, as is well-known, for a condition number $C N$ one can expect to $\operatorname{lose} O\left(\log _{10}[C N]\right)$ digits of precision in computing the weights [36].

${ }^{34}$ We set $T_{0}=0.25 \mathrm{~m}, q=0$, and $\xi_{1}=\Delta \xi_{x}=\cos \gamma^{+} \Delta \xi_{x}^{t^{\prime}}=\pi / \Delta x$ for all cases in both figures.
} 
To confirm that the system matrix ill-conditioning is not due to the complex-plane generalization of the new MWA, in Figure 4a we show the two-norm condition number for $\gamma^{+}=\pi / 4$ while in Figure $4 \mathrm{~b}$ we show, for the same four $\mathbf{r}-\mathbf{r}^{\prime}$ geometries, the conditioning for $\gamma^{+}=0$ (i.e. as if we performed the standard, real-axis MWA from [20]).

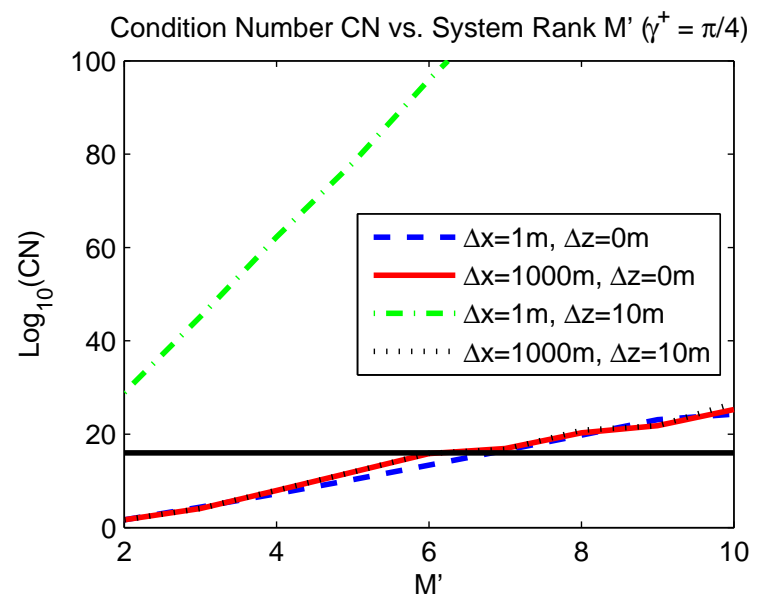

(a)

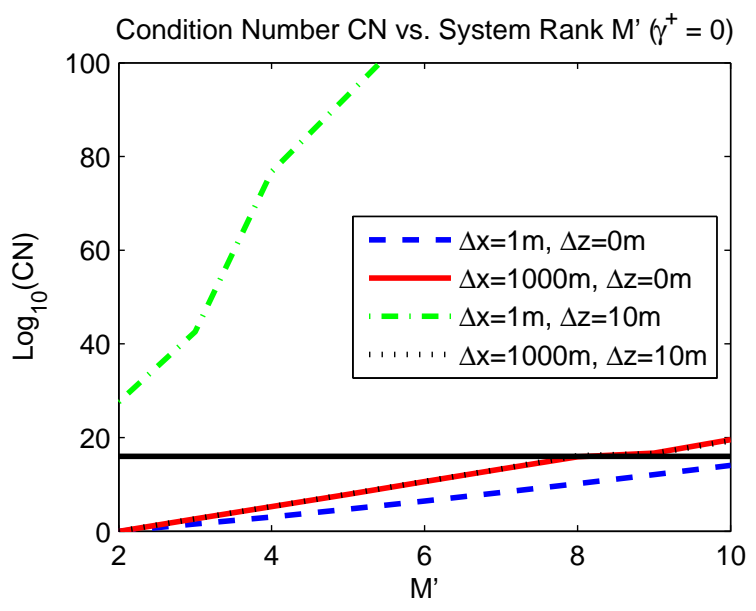

(b)

Figure 4: Base-10 logarithm of the two-norm system matrix condition number used to compute the new MWA weights, as specified in (4.11) and [20], for Figures 4a and 4b (resp.). The vertical axis displays the number of digits of precision lost in the weights, when numerically computing them, due to the conditioning of 4.10 . The solid horizontal curve corresponds to $\log _{10}(C N)=16$; weights arising as solutions to a rank- $M^{\prime}$ linear system with condition number greater than this are expected to be just numerical "noise" when computed using IEEE double-precision arithmetic.

One readily observes from Figure 4 that accurate weight computation is unrealistic as $M^{\prime}$ increases; in fact, the situation is downright prohibitive for an adaptive MWA implementation (e.g. [19]). Even for the best-conditioned geometry (i.e. $\left.\left(x-x^{\prime}, z-z^{\prime}\right)=(1,0) \mathrm{m}\right)$, one cannot realistically expect even a single digit of precision in the weights for $M^{\prime}$ equalling or exceeding approximately seven and ten in Figures $4 \mathrm{a}$ and $4 \mathrm{~b}$ (resp.), as can be seen from the intersection of the corresponding curves in Figures $4 \mathrm{a} 4 \mathrm{~b}$ with the solid horizontal curve corresponding to $\log _{10}(C N)=16$. As a result, we choose the second proposed MWA formulation, based on the MMA [18], for computing validation results in Section 5] Based on our previous work using the standard, real-axis MMA [19] for environments containing high loss and conductively-uniaxial layers, one can expect its success in again producing high-precision results. Indeed, the validation results in Section 5 speak to this effec 35

\section{Results and Discussion}

In this section we exhibit validation results in scenarios involving the modeling of induction sondes for geophysical prospection of hydrocarbons (i.e. induction well logging [5]). Previously, we demonstrated numerous simulated resistivity logs pertaining to environments containing a combination of isotropic and reciprocal, electrically uniaxial media [37] as probed by longitudinally-oriented induction sondes [19]. For those case studies, the adaptive, real-axis MMA was successfully incorporated into our algorithm to yield high-precision results exhibiting excellent agreement with data from previous literature [2]-4].

Herein, we exhibit a case study involving a near-horizontal tool orientation where the tool axis dip angle $\alpha=89^{\circ}$, tool axis strike angle $\beta=0^{\circ} 36$, and source-observer separation $L_{\text {tool }}=\left|\mathbf{r}-\mathbf{r}^{\prime}\right|=40^{\prime}=1.016 \mathrm{~m}$, corresponding to

\footnotetext{
${ }^{35}$ Due to the intricate, recursively-related nature of the MMA weight set tiers, a straightforward definition and analysis of the conditioning of the problem related to procuring these weights proves elusive and therefore is not pursued here. Rather, its use herein is strictly based on, and justified by, its empirically-demonstrated efficacy in [18, 19].

${ }^{36}$ The tool thus rotates and is confined within the $x z$ plane [7].
} 
a source-observer depth separation $\left|z-z^{\prime}\right|=L_{\text {tool }} \cos \alpha \sim 17.7 \mathrm{~mm}$. Consequently, this study serves to validate the efficacy of our new algorithm and its ability to impart absolute, exponential-cum-algebraic convergence on Fourier double-integrals like (1.1) even for the traditionally prohibitive regime $\left|z-z^{\prime}\right| \ll 1$. Furthermore, to exemplify the general-purpose nature of our new algorithm in regards to the media present, we generate synthetic resistivity logs for a two-layer, planar-stratified environment containing reciprocal, electrically biaxial medie ${ }^{37}$ In this scenario, wherein all four characteristic plane wave modes in the anisotropic layer containing $\mathbf{r}$ can (in general) contribute to the observed field, the definition of an exact " $\Delta z$ " and thus CPP is ill-defined (see Section 3). Therefore, this set of results also justifies our retaining the MWA's robust environment/source-observer geometry convergence acceleration characteristic, yielding an overall robust and rapid electromagnetic field solution method.

Note that save for Figure 8f] there exists strong agreement across the full logging path in each plot. Even for Figure 8f], with some discrepancy in the upper half-space $D>0$, overall there is strong qualitative agreement and (in the bottom half-space) quantitative agreement to $\sqrt{38}$

\footnotetext{
${ }^{37}$ For Figures 5.8 the frequency of operation is $f=2 \mathrm{MHz}$, the interface is located at $z_{B}=0 \mathrm{~m}$, and the resistivity tensor for layer $n$ (with respect to the standard Earth system) is described in the figure headings by the diagonal matrix $R_{n}=\left[R_{x^{\prime} x^{\prime}}, R_{y^{\prime} y^{\prime}}, R_{z^{\prime} z^{\prime}}\right]=\operatorname{diag}\left[R_{x^{\prime} x^{\prime}}, R_{y^{\prime} y^{\prime}}, R_{z^{\prime} z^{\prime}}\right]$.

${ }^{38}$ For the figures shown below, the material formation parameter labeling is reversed versus the labeling in the reference paper such that the material scenario $\left\{R_{1}=[100,200,500] \Omega \mathrm{m}, R_{2}=[1,2,5] \Omega \mathrm{m}\right\}$ corresponds to $\left\{R_{1}=[100,50,500] \Omega \mathrm{m}, R_{2}=[1,0.5,5] \Omega \mathrm{m}\right\}$ in $[1]$ and vice versa. Due to the strong agreement between the simulation data produced here and by the reference source after this labeling reversal, we suspect this apparent data discrepancy is attributed to a simple typographical error on the part of the authors of [1].
} 


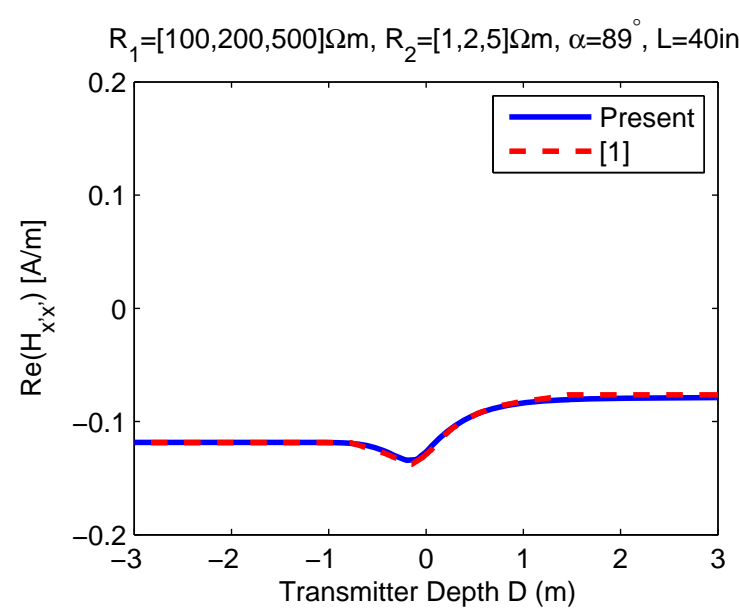

(a)

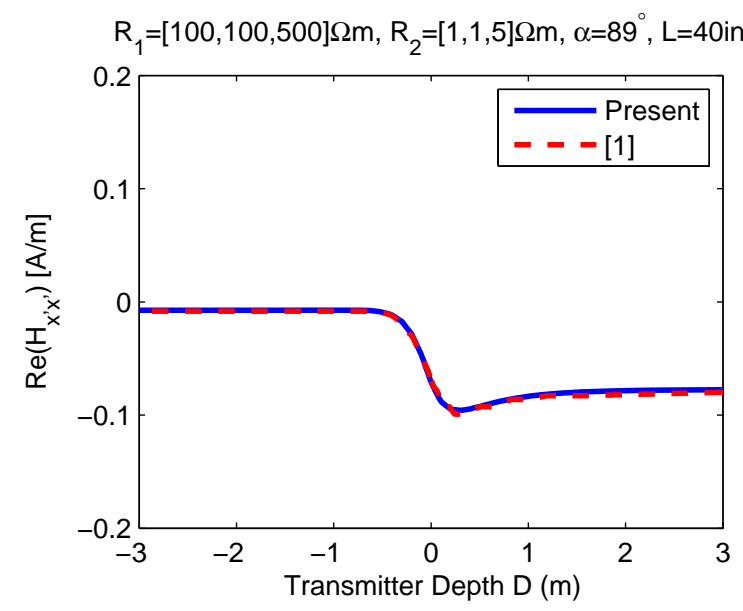

(c)

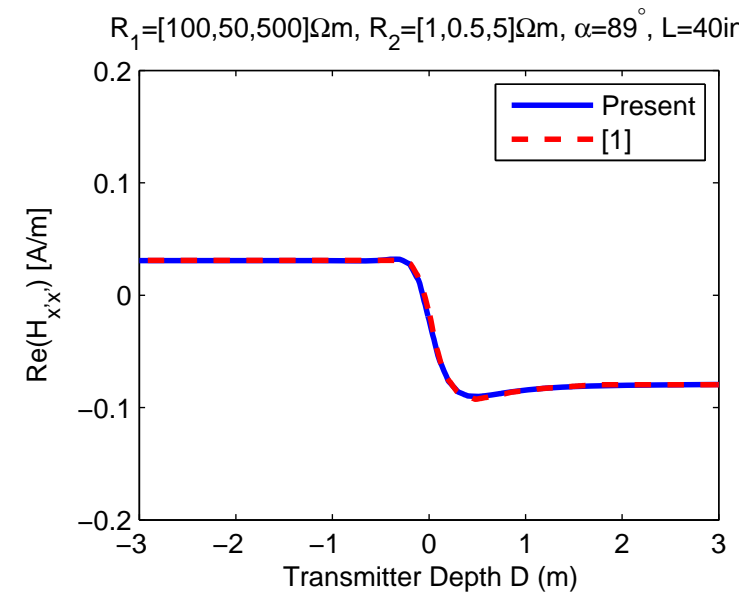

(e)

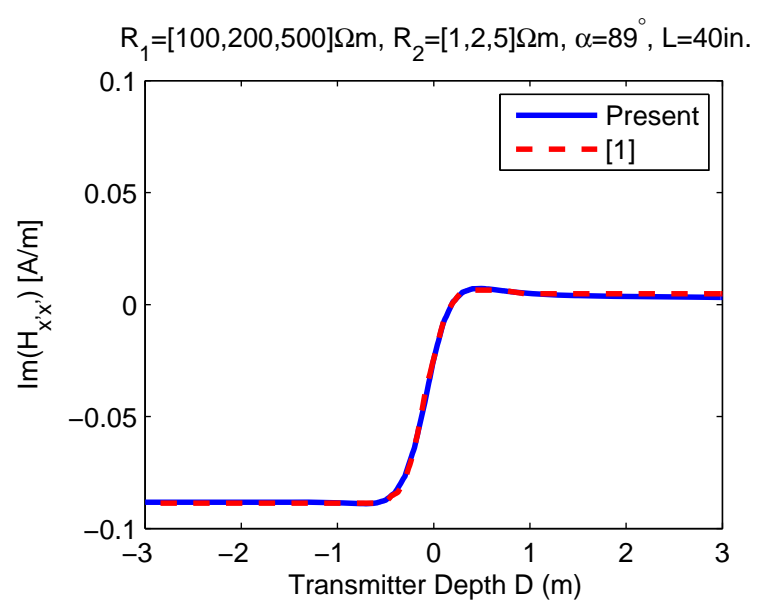

(b)

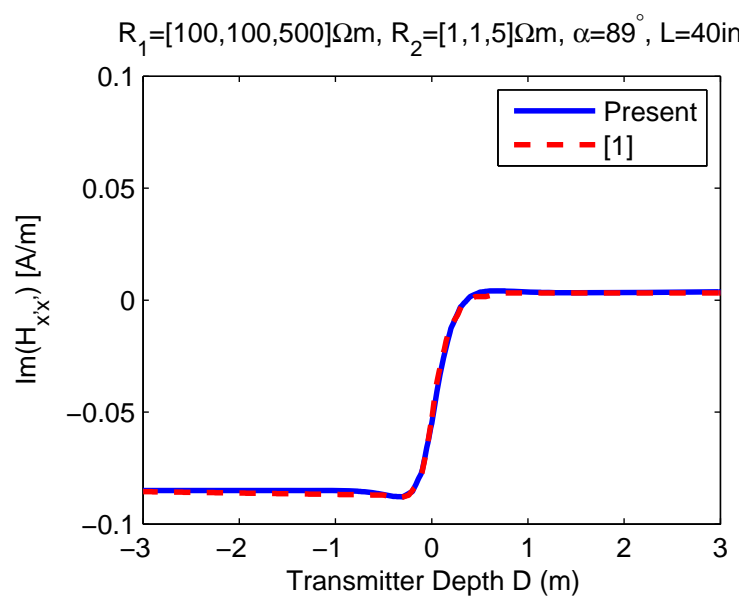

(d)

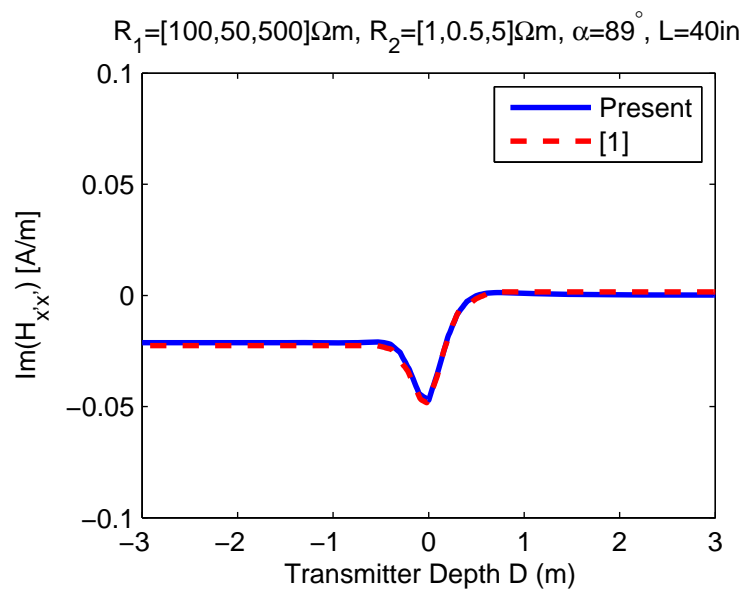

(f)

Figure 5: Comparison of simulated magnetic field $H_{x^{\prime} x^{\prime}}$ with Figure 4 of [1]. 


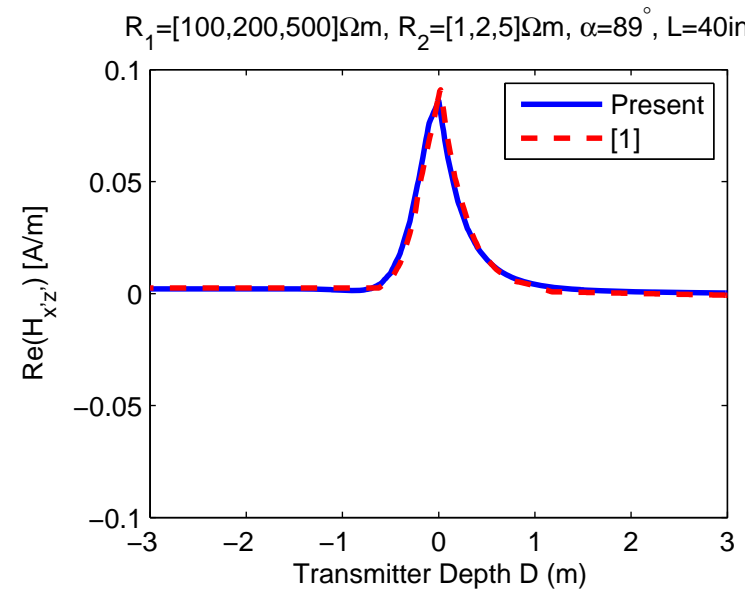

(a)

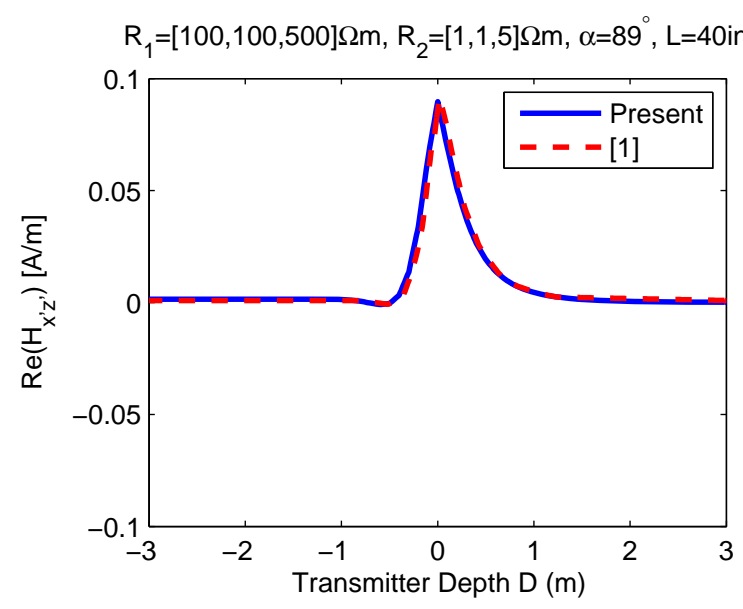

(c)

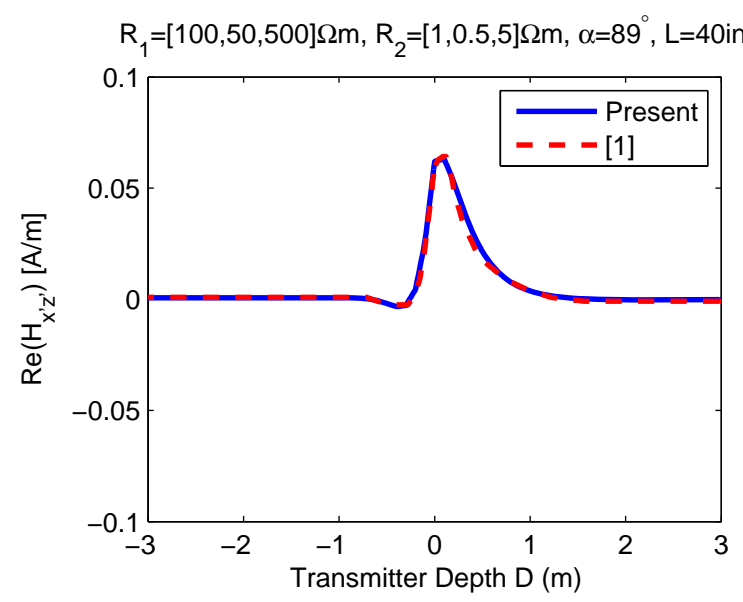

(e)

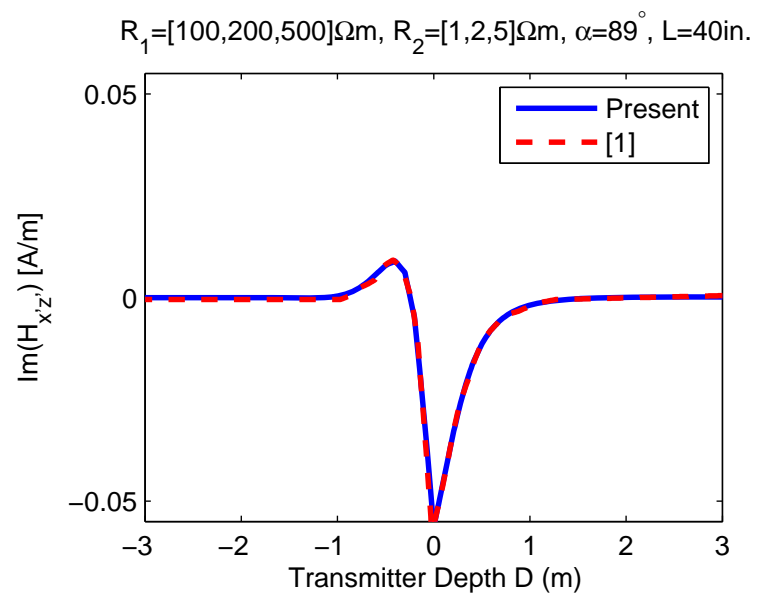

(b)

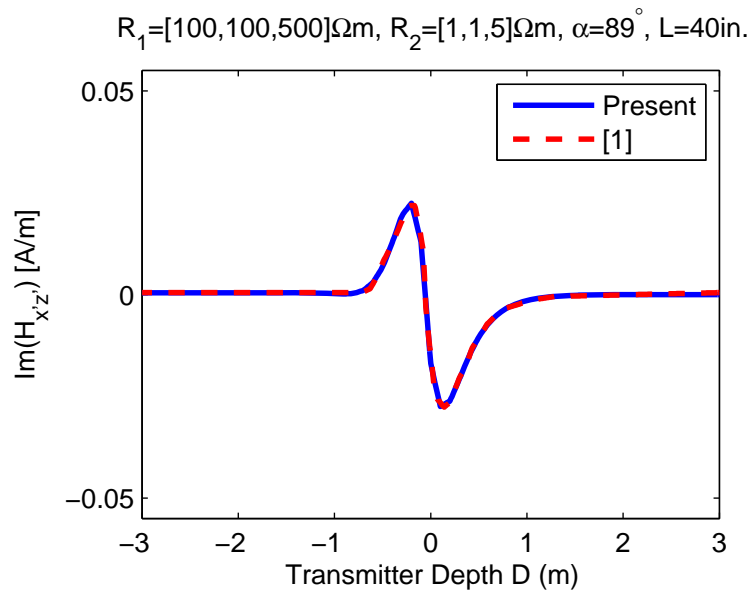

(d)

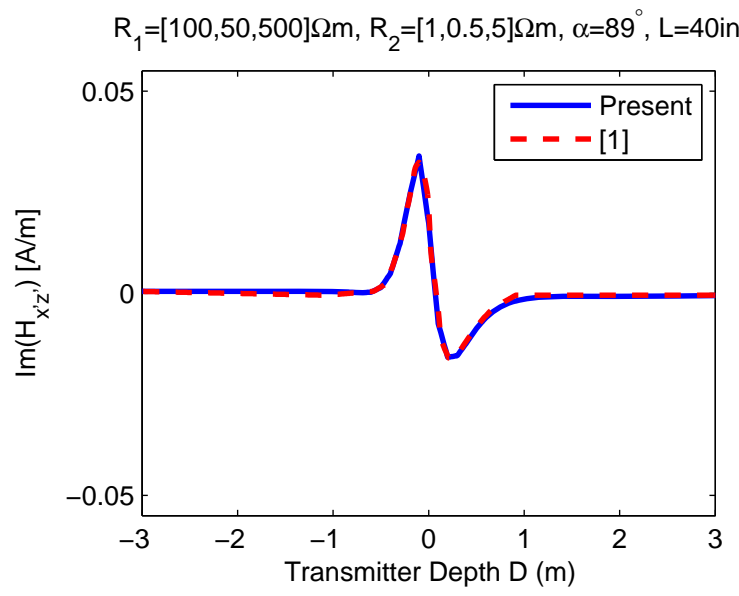

(f)

Figure 6: Comparison of simulated magnetic field $H_{x^{\prime} z^{\prime}}$ with Figure 4 of [1]. 


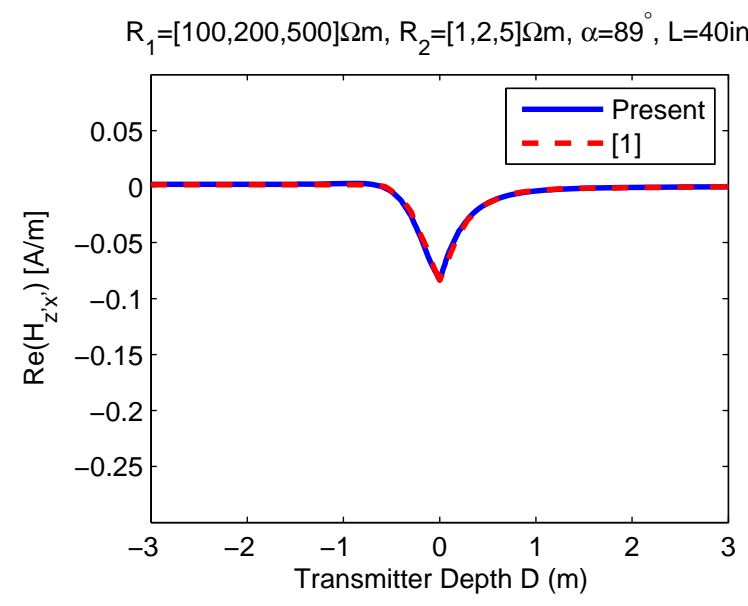

(a)

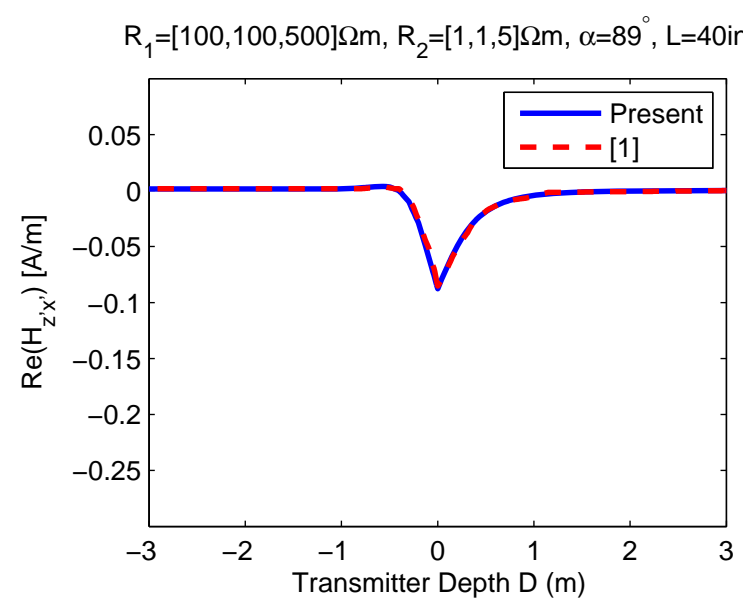

(c)

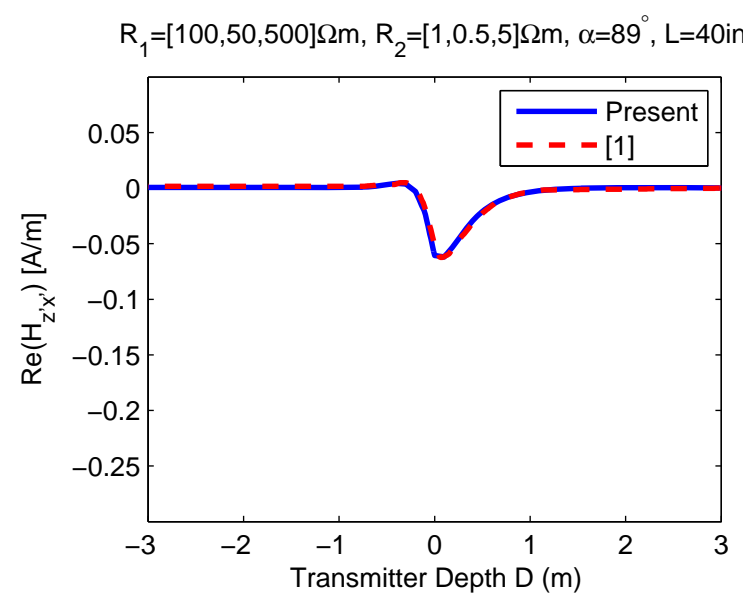

(e)

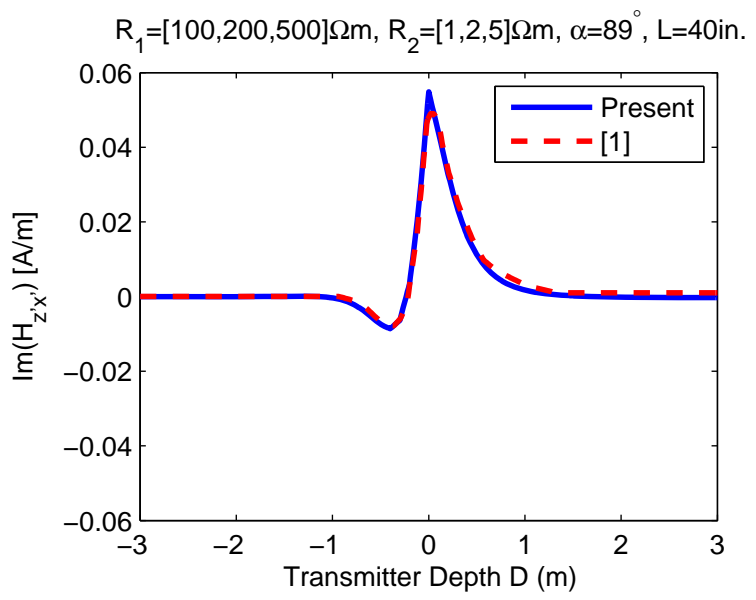

(b)

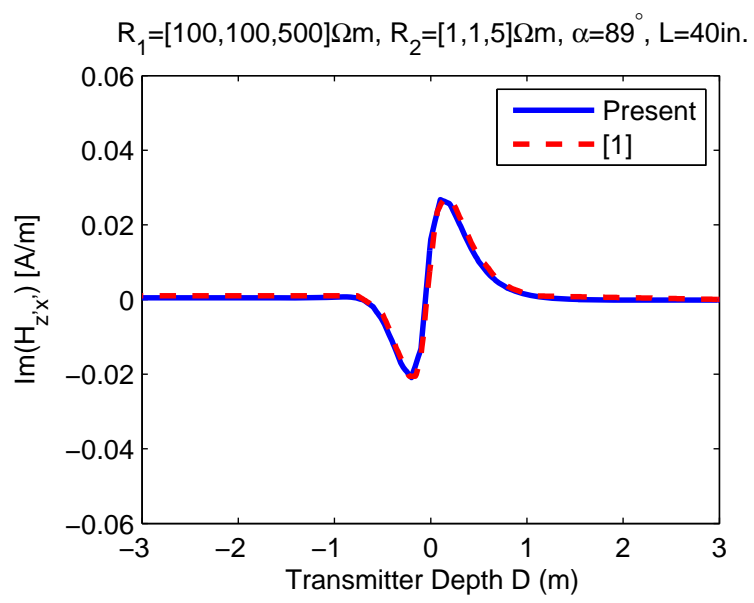

(d)

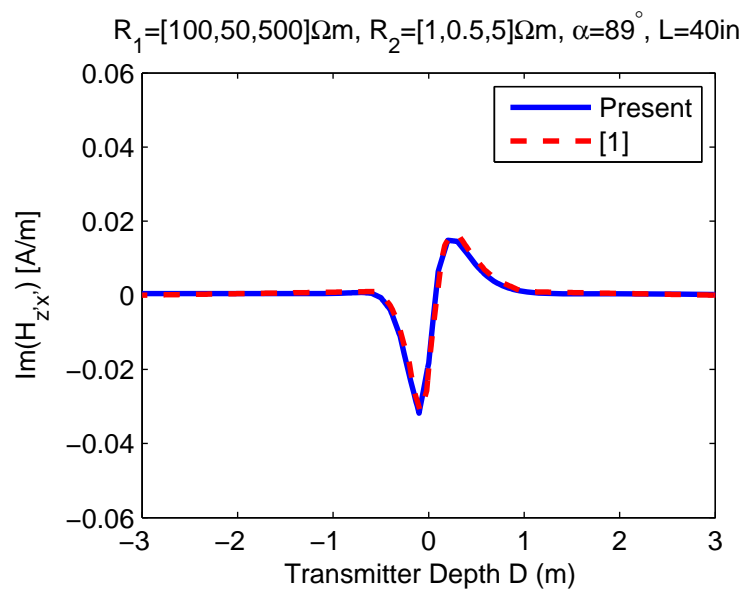

(f)

Figure 7: Comparison of simulated magnetic field $H_{z^{\prime} x^{\prime}}$ with Figure 4 of [1]. 


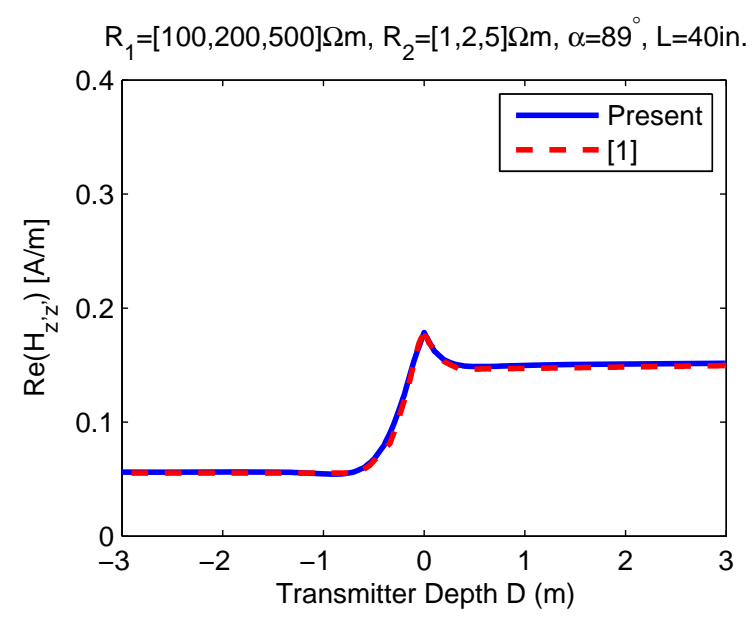

(a)

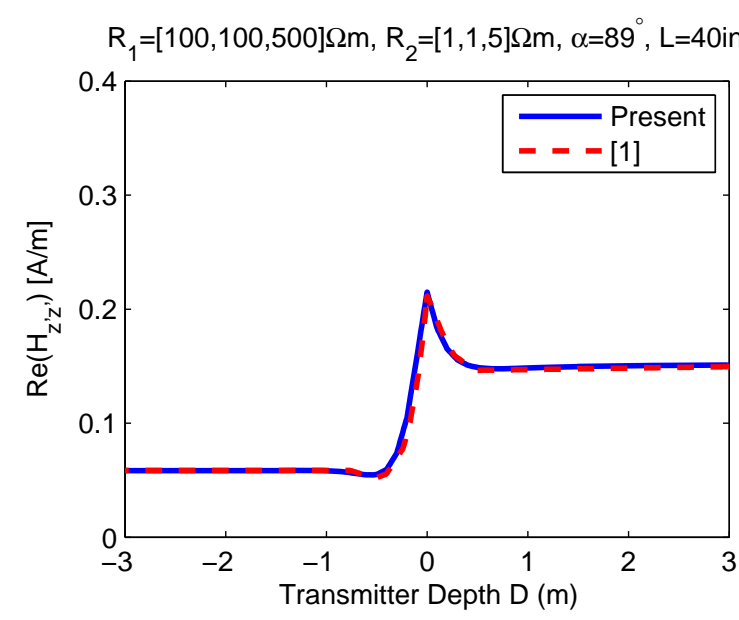

(c)

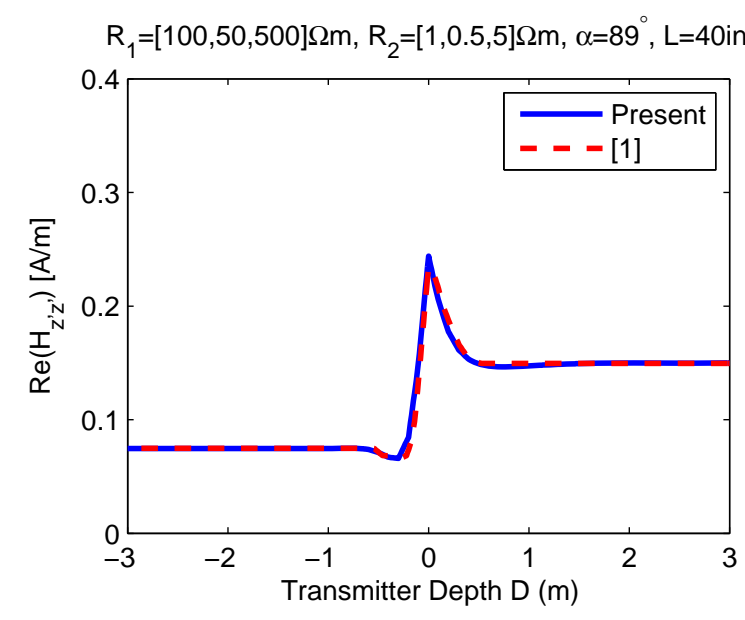

(e)

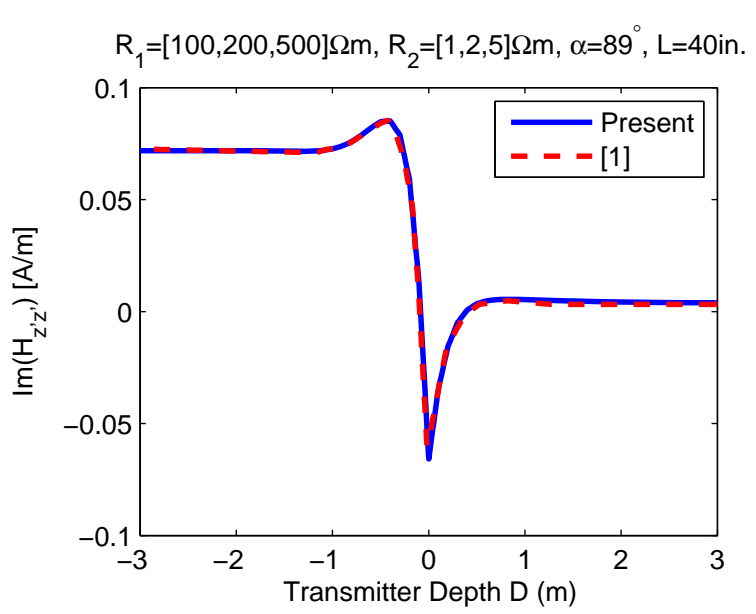

(b)

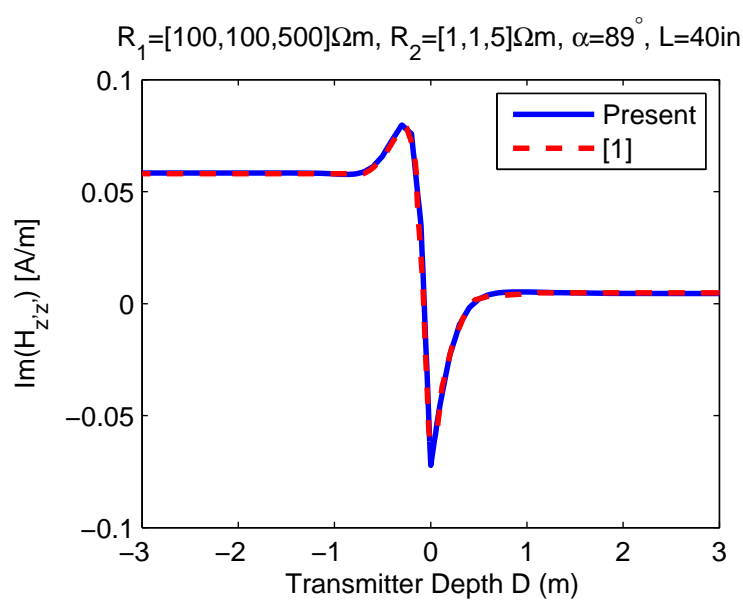

(d)

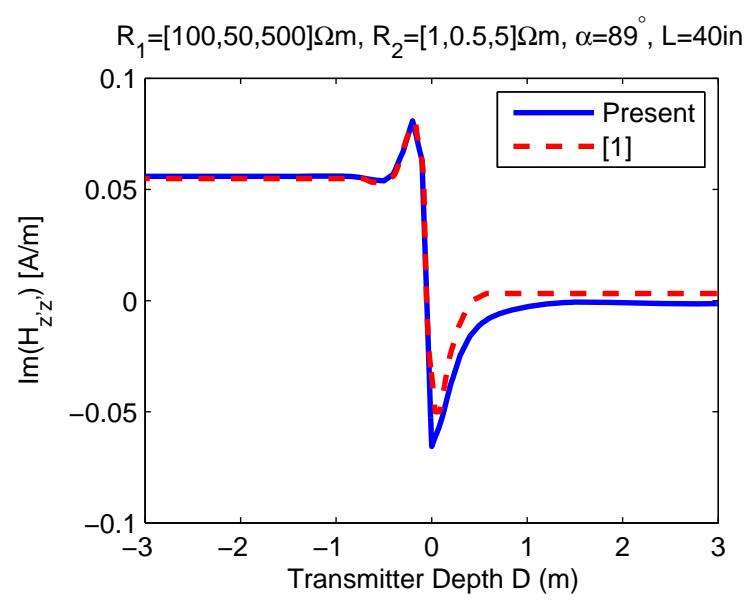

(f)

Figure 8: Comparison of simulated magnetic field $H_{z^{\prime} z^{\prime}}$ with Figure 4 of [1]. 


\section{Convergence Characteristics}

To characterize our numerical formulation's ability to converge towards the field solution, we present two case studies concerning the $z$-directed electric field component $E_{z}$ produced by a $z$-directed electric dipole radiating at $f=2 \mathrm{MHz}$ in free space. The first case comprises a benign scenario in which $x-x^{\prime}=y-y^{\prime}=z-z^{\prime}=1 \mathrm{~m}$, while the second case represents a very challenging scenario wherein $x-x^{\prime}=500 \mathrm{~m}$ and $y-y^{\prime}=z-z^{\prime}=0 \mathrm{~m}$. The latter scenario's prohibitive challenges, when using a standard numerical integration method, are that the integrand (1) oscillates on the order of 500/ $\sqrt{2}$ times more rapidly than the integrand in case on ${ }^{39}$ and (2) exhibits absolutely $n o$ exponential decay due to the annihilation of the $\exp \left(i \tilde{k}_{z}^{+}\left|z-z^{\prime}\right|\right)$-type factors. If we were to use a traditional numerical integration methodology, we emphasize that one would obtain a divergent result.

For each case, we present results related to the Region III field contribution (see Figure 3). Since one cannot obtain a closed-form solution to this field contribution, reference field values from which one measures relative accuracy must be appropriately chosen; their computation details are provided in Figure 9 below. As in [17--20], we assume the integrand is well-behaved in Region III and thus do not perform adaptive interval sub-division. Instead, we set the $k_{x}$ and $k_{y}$ plane extrapolation region interval lengths as per Section 3 and examine the accuracy versus (1) the number of extrapolation region intervals employed $(B)$ and (2) the Legendre-Gauss quadrature order used $(L G Q)$ to integrate each interval40

Our comments on the relative importance of aliasing and truncation error are analogous to [19]: Up to approximately $B=3$ the truncation error dominates the total relative error, while using more than approximately $B=6$ or 7 intervals effects no noticeable decrease in the error for a fixed $L G Q$. Beyond this point aliasing error dominates the total relative error, which is evidenced by the error decreasing versus increasing $L G Q$ but remaining flat versus increasing $B$. However, we notice the following two remarkable characteristics about the algorithm's convergence for case two:

1. The $L G Q=30$ curve reaches within $25 \mathrm{~dB}$ of case one's $L G Q=30$ curve despite representing a scenario wherein the field solution would ordinarily have diverged using standard numerical integration techniques.

2. Despite this case representing a far more prohibitive scenario (if traditionally evaluated) versus case two presented in [19], wherein $\mathbf{r}-\mathbf{r}^{\prime}=(500,500,1) \mathrm{m}$, at $B=10$ the $L G Q=30$ curve here levels off at an error approximately $23 \mathrm{~dB}$ lower than its case two counterpart in [19].

Note that (akin to, and for the same reasons stated in, [19]) relative errors below -150dB were coerced to $-150 \mathrm{~dB}$.

Since the pre-extrapolation region formulation in this paper is not radically different from that in [19], we expect similar convergence characteristics when using the trapezoidal detour (versus those presented in [19]) and thus omit the Region I convergence study for brevity. Furthermore, the Region IIa/IIb convergence studies are omitted as well since the field convergence results would be affected by the algorithm's handling of both the pre-extrapolation and extrapolation region sections of the $k_{x}$ and $k_{y}$ plane integration paths. Equivalently, presenting information on the Region I and Region III field convergence characteristics sheds insight into the Region IIa/IIb convergence characteristics. This is because if the respective algorithms handling the Region I and Region III integrations robustly yield accurate, rapidly convergent results, one can expect similar behavior for the Region IIa/IIb results.

\footnotetext{
${ }^{39}$ Recall the azimuthal coordinate rotation performed such that in the rotated basis $x-x^{\prime}=y-y^{\prime} \geq 0$.

${ }^{40} B$ intervals are used in both the $k_{x}^{\prime}>0$ and $k_{x}^{\prime}<0$ integration path half-tails; the same applies for the $k_{y}$ path half-tails.
} 


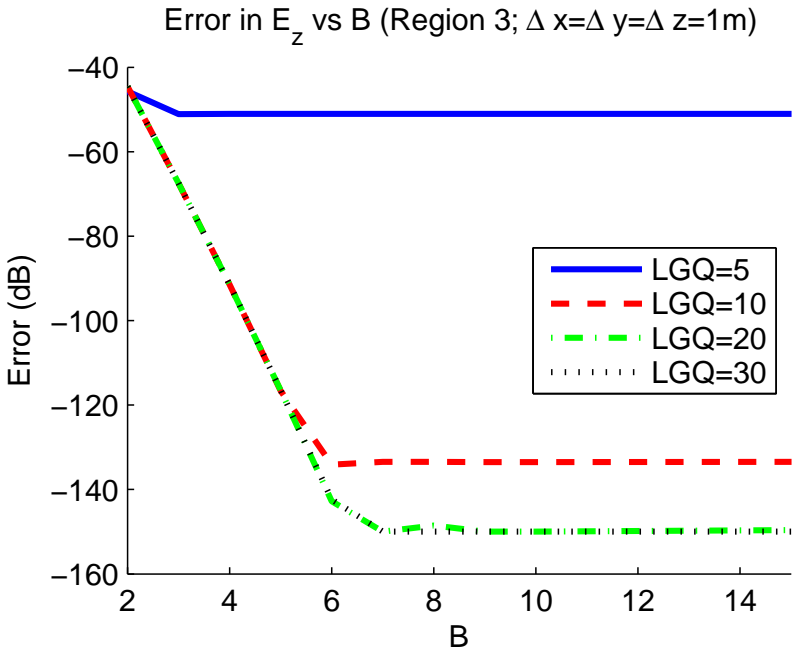

(a)

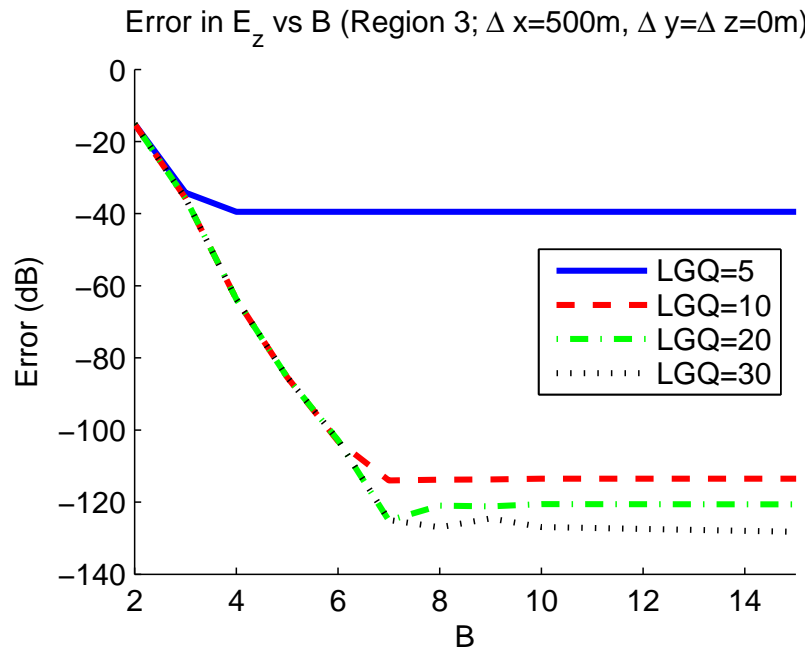

(b)

Figure 9: Convergence towards the solution comprising the $E_{z}$ contribution from Region III. The reference field values are computed using $L G Q=30$ and $B=500$ for both figures.

\section{Conclusion}

In this work, we have presented a novel integration scheme composed of (1) a complex-plane, adaptive/errorcontrolling extension to the standard real-axis MMA in conjunction with (2) a more robust pre-extrapolation region integration path to effect fast, absolute, and exponential-cum-algebraic convergence of Fourier- and F-H-type integral transforms such as (1.1)-(1.4). Due to combining the detour with the MMA and its robust algebraic convergence acceleration characteristic, this is indeed the case irrespective of the source-observer geometry and loss/anisotropy characteristics of the stratified media present. Furthermore, this is accomplished without the added complication of having to separately account for slab/interface mode contributions whose poles may be crossed when otherwise deforming to more well-known, rapidly-convergent paths such as the SDP [16, 27], resulting in a numerically robust and easily-implemented integration methodology.

The algorithm's ability to accurately simulate the observed fields for classically "worst-case" scenarios $0 \leq \mid z-$ $z^{\prime} \mid \ll 1$, and that too in complex, planar-stratified environments containing biaxial-conductive media, has been verified through numerous validation checks against [1]. Finally, the algorithm's convergence characteristics in the stronglyevanescent spectral zone have been explored, analyzed, and shown to be superior compared to an older methodology exhibited in [19] that was based on an adaptive extension to the real-axis MMA.

We conclude that the present algorithm's robustness with respect to source-observer geometries and medium types present, as well as its straight-forward nature and ease of implementation, makes it very useful for the analysis of electromagnetic wave propagation and scattering in multi-layered environments containing media of arbitrary anisotropy and loss.

\section{Acknowledgements}

We thank Halliburton Energy Services for the permission to publish this work. We also acknowledge partial support from the Ohio Supercomputing Center under Grant PAS-0061. 


\section{References}

\section{References}

[1] S. Davydycheva, T. Wang, Modeling of Electromagnetic Logs in a Layered, Biaxially Anisotropic Medium, in: SEG Annual Meeting, 2011, pp. 494-498.

[2] B. Wei, T. Wang, Y. Wang, Computing the Response of Multi-Component Induction Logging in Layered Anisotropic Formation by the Recursive Matrix Method with Magnetic-Current-Source Dyadic Green's Function, Chinese Journal of Geophysics 52 (2009) $1350-1359$.

[3] B. I. Anderson, T. D. Barber, S. C. Gianzero, The Effect of Crossbedding Anisotropy on Induction Tool Response, in: SPWLA 39th Annual Logging Symposium, 1998, pp. 1-14.

[4] A. Howard Jr., Petrophysics of Magnetic Dipole Fields in an Anisotropic Earth, IEEE Transactions on Antennas and Propagation 48 (2000) 1376-1383.

[5] M. Zhdanov, W. Kennedy, E. Peksen, Foundations of Tensor Induction Well-Logging, Petrophysics 42 (2001) 588-610.

[6] H. Wang, H. Tao, J. Yao, G. Chen, S. Yang, Study on the Response of a Multicomponent Induction Logging Tool in Deviated and Layered Anisotropic Formations by using Numerical Mode Matching Method, Chinese Journal of Geophysics 51 (2008) 1110-1120.

[7] J. Moran, S. Gianzero, Effects of Formation Anisotropy on Resistivity-Logging Measurements, Geophysics 44 (1979) $1266-1286$.

[8] M. Paulus, P. Gay-Balmaz, O. J. F. Martin, Accurate and Efficient Computation of the Green's Tensor for Stratified Media, Phys. Rev. E 62 (2000) 5797-5807.

[9] D. Pozar, V. Sanchez, Magnetic Tuning of a Microstrip Antenna on a Ferrite Substrate, Electronics Letters 24 (1988) 729-731.

[10] D. M. Pozar, Radiation and Scattering from a Microstrip Patch on a Uniaxial Substrate, IEEE Transactions on Antennas and Propagation 35 (1987) 613-621.

[11] M. Jain, J. K. Lotsberg, J. J. Stamnes, Comparisons of Exact and Paraxial Intensities of Electromagnetic Waves Focused into Uniaxial Crystals, Journal of Optics A: Pure and Applied Optics 8 (2006) 709.

[12] M. Jehle, M. Ruegg, L. Zuberbuhler, D. Small, E. Meier, Measurement of Ionospheric Faraday Rotation in Simulated and Real Spaceborne SAR Data, IEEE Transactions on Geoscience and Remote Sensing 47 (2009) 1512-1523.

[13] S. Lambot, E. Slob, H. Vereecken, Fast Evaluation of Zero-Offset Green's Function for Layered Media with Application to GroundPenetrating Radar, Geophysical Research Letters 34 (2007).

[14] S. Lambot, E. C. Slob, M. Vanclooster, H. Vereecken, Closed Loop GPR Data Inversion for Soil Hydraulic and Electric Property Determination, Geophysical Research Letters 33 (2006).

[15] J. R. Mosig, F. E. Gardiol, A Dynamical Radiation Model for Microstrip Structures, Advances in Electronics and Electron Physics 59 (1982) $139-237$.

[16] W. C. Chew, Waves and Fields in Inhomogeneous Media, Van Nostrand Reinhold, 1990, pp. 45-160.

[17] K. A. Michalski, Application of the Complex Image Method to Electromagnetic Field Computation in Planar Uniaxial Multilayers, in: Workshop on Integral Techniques for Electromagnetics, Lausanne, Switzerland, 2007.

[18] K. A. Michalski, Extrapolation Methods for Sommerfeld Integral Tails, IEEE Transactions on Antennas and Propagation 46 (1998) 14051418.

[19] K. Sainath, F. L. Teixeira, B. Donderici, Robust Computation of Dipole Electromagnetic Fields in Arbitrarily-Anisotropic, Planar-Stratified Environments, 2013. ArXiv:1309.7952v1.

[20] J. Mosig, The Weighted Averages Algorithm Revisited, IEEE Transactions on Antennas and Propagation 60 (2012) $2011-2018$.

[21] R. Golubovic, A. Polimeridis, J. Mosig, Efficient Algorithms for Computing Sommerfeld Integral Tails, IEEE Transactions on Antennas and Propagation 60 (2012) 2409-2417.

[22] A. Alparslan, M. Aksun, K. Michalski, Closed-Form Green's Functions in Planar Layered Media for All Ranges and Materials, IEEE Transactions on Microwave Theory and Techniques 58 (2010) 602-613.

[23] A. Caboussat, G. Miers, Numerical Approximation of Electromagnetic Signals Arising in the Evaluation of Geological Formations, Computers \& Mathematics with Applications 59 (2010) 338 - 351.

[24] H. H. Homeier, Scalar Levin-Type Sequence Transformations, Journal of Computational and Applied Mathematics 122 (2000) 81 - 147.

[25] J. Mosig, A. Melcon, Green's Functions in Lossy Layered Media: Integration along the Imaginary Axis and Asymptotic Behavior, IEEE Transactions on Antennas and Propagation 51 (2003) 3200-3208.

[26] J. Mosig, F. Gardiol, Analytical and Numerical Techniques in the Green's Function Treatment of Microstrip Antennas and Scatterers, IEE Proceedings H: Microwaves, Optics and Antennas 130 (1983) 175-182.

[27] B. Hu, W. Chew, Fast Steepest Descent Path Algorithm for Electromagnetic Scattering Problems in Layered Medium, in: IEEE Antennas and Propagation Society International Symposium 1999, volume 2, 1999, pp. 1182-1185 vol.2. doi 10.1109/APS.1999.789524

[28] W. C. Chew, Waves and Fields in Inhomogeneous Media, Van Nostrand Reinhold, 1990, pp. 161-209.

[29] H. Moon, F. L. Teixeira, B. Donderici, Stable Pseudoanalytical Computation of Electromagnetic Fields from Arbitrarily-Oriented Dipoles in Cylindrically Stratified Media, 2013. ArXiv:1308.3179v1.

[30] J. R. Mosig, Integral equation technique, in: T. Itoh (Ed.), Numerical Techniques for Microwave and Millimeter Wave Passive Structures, Wiley, New York, NY, 1989, pp. 133-213.

[31] W. C. Chew, Waves and Fields in Inhomogeneous Media, Van Nostrand Reinhold, 1990, pp. 1-44.

[32] K. T. R. Davies, Complex-Plane Methods for Evaluating Highly Oscillatory Integrals in Nuclear Physics. ii, Journal of Physics G: Nuclear Physics 14 (1988) 973.

[33] Fubini's Theorem, http://en.wikipedia.org/wiki/Fubini'_s_theorem/2013. [Online]. Accessed August $25,2013$.

[34] W. C. Chew, Waves and Fields in Inhomogeneous Media, Van Nostrand Reinhold, 1990, pp. $375-428$.

[35] System of Linear Equations, http://en.wikipedia.org/wiki/System_of_linear_equations/. 2013. [Online]. Accessed August $11,2013$.

[36] L. N. Trefethen, D. Bau, Numerical Linear Algebra, SIAM: Society for Industrial and Applied Mathematics, 1997. 
[37] L. B. Felsen, N. Marcuvitz, Radiation and Scattering of Waves, Electromagnetic Waves, IEEE Press, Piscataway, NJ, 1994, pp. 740-820. 


\section{Appendix A. Appendix: Definitions and Conventions}

We state the following regarding notation used in this paper:

1. $i$ is the unit-magnitude imaginary number.

2. The time-harmonic field convention used and suppressed throughout this paper is $\exp (-i \omega t)$, where $\omega$ is the angular frequency at which the source distribution radiates.

3. $c$ is the speed of light in free space.

4. $k_{o}=\omega / c$ is the characteristic wave number of free space.

5. $\mathbf{r}=(x, y, z)$ denotes the observer location, while $\mathbf{r}_{t}=(x, y)$ denotes the transverse observer location with magnitude $\rho=\sqrt{x^{2}+y^{2}}$.

6. $\mathbf{r}^{\prime}=\left(x^{\prime}, y^{\prime}, z^{\prime}\right)$ denotes the source location, while $\mathbf{r}_{t}^{\prime}=\left(x^{\prime}, y^{\prime}\right)$ denotes the transverse source location with magnitude $\rho^{\prime}=\sqrt{x^{\prime 2}+y^{\prime 2}}$.

7. $\mathbf{k}=\left(k_{x}, k_{y}, k_{z}\right)$ denotes the wave vector, while $\mathbf{k}_{t}=\left(k_{x}, k_{y}\right)$ denotes the transverse wave vector with complex amplitude $k_{\rho}=\sqrt{k_{x}^{2}+k_{y}^{2}}$. It is implicitly understood that one evaluates $\sqrt{k_{x}^{2}+k_{y}^{2}}$ such that $H_{n}^{(1)}\left(k_{\rho}\left|\rho-\rho^{\prime}\right|\right)$ (see definition 10 below) corresponds to an exponentially decaying function versus increasing $\left|\rho-\rho^{\prime}\right|$.

8. The axial wave number component for the $n$th characteristic mode supported in layer $M, \tilde{k}_{M, n z}$, is coupled to the transverse wave numbers $k_{x}$ and $k_{y}$ via the $n$th mode's dispersion relation.

9. The up-going or down-going mode's axial wave number component in some isotropic layer is denoted by $\tilde{k}_{z}^{+}$or $\tilde{k}_{z}^{-}$(resp.) when the particular layer is not critical to understanding the discussion.

10. $H_{n}^{(1)}\left(k_{\rho}\left|\rho-\rho^{\prime}\right|\right)$ is the order- $n$ Hankel function of the first kind, corresponding to an out-going cylindrical wave.

11. Quantities dependent only on one or more spectral variables $\left\{k_{x}, k_{y}, k_{z}\right\}$ are denoted spectral quantities and are distinguished with an over-tilde (e.g. $\tilde{f}\left(k_{x}, k_{y}, k_{z}\right)$ ).

12. Quantities dependent on $\mathbf{k}_{t}, z$, and $z^{\prime}$ are denoted mixed-domain quantities and have no over-symbol.

13. Numbers expressed as $\left(\begin{array}{l}A \\ B\end{array}\right)$ correspond to the binomial coefficients.

14. $\epsilon_{m, r}$ and $\mu_{m, r}$ represent the relative electric permittivity (including conductive and polarization losses) and relative magnetic permeability of isotropic layer $m$.

15. $\operatorname{Re}\left[k_{x}\right]$ and $\operatorname{Im}\left[k_{x}\right]$ are used interchangeably with $k_{x}^{\prime}$ and $k_{x}^{\prime \prime}$ (resp.) to denote the real and imaginary part of $k_{x}$ (resp.). Analogous definitions apply for $k_{y}$ and other complex-valued quantities. 